\title{
Ruled quartic surfaces, models and classification
}

\author{
Irene Polo-Blanco • Marius van der Put • Jaap Top
}

Received: 15 April 2009 / Accepted: 12 April 2010 / Published online: 30 April 2010

(C) The Author(s) 2010. This article is published with open access at Springerlink.com

\begin{abstract}
New historical aspects of the classification, by Cayley and Cremona, of ruled quartic surfaces and the relation to string models and plaster models are presented. In a "modern' treatment of the classification of ruled quartic surfaces the classical one is corrected and completed. The string models of Series XIII of some ruled quartic surfaces (manufactured by L. Brill and by M. Schilling) are based on a result of Rohn concerning curves in $\mathbb{P}^{1} \times \mathbb{P}^{1}$ of bi-degree $(2,2)$. This is given here a conceptional proof.
\end{abstract}

Keywords Ruled surface · Quartic suface · Grassmann variety · Dual surface ·

Reciprocal surface

Mathematics Subject Classification (2000) $\quad 14-03 \cdot 01-02 \cdot 14 \mathrm{~J} 26 \cdot 14 \mathrm{M} 15 \cdot 14 \mathrm{~N} 25 \cdot 32 \mathrm{~S} 25$

\section{o Motivation and Introduction}

\subsection{The models of K. Rohn}

The series of ten string models of ruled quartic surfaces, present at some mathematical institutes (for instance at the department of mathematics in Groningen) is the direct motivation for this paper. This Series XIII, first produced by L. Brill in 1886 and later by Martin Schilling, is based upon a paper of K. Rohn [13] containing a classification of ruled quartic surfaces

I. Polo-Blanco

Department Matesco, University of Cantabria, Avda de los Castros s/n, 39005 Santader, Spain e-mail: irene.polo@unican.es

M. van der Put $\cdot$ J. Top $(\bowtie)$

Department of Mathematics, University of Groningen, P.O. Box 407, 9700 AK Groningen,

The Netherlands

e-mail: j.top@rug.nl

M. van der Put

e-mail: mvdput@math.rug.nl 
over $\mathbb{C}$ and $\mathbb{R}$. As a start we will explain some of the geometry of these models. The classical work of Cayley, Cremona et al. on ruled quartic surfaces and a modern viewpoint will be discussed in later sections.

Classically, a ruled surface $S$ is a reduced, irreducible surface in projective space $\mathbb{P}^{3}$ (say, over the complex numbers) which is the union of the (straight) lines on it. We consider $S$ of degree 4 and omit cones over a plane quartic curve. A $(2,2)$ correspondence is a curve $\Gamma \subset \mathbb{P}^{1} \times \mathbb{P}^{1}$, defined by an irreducible bi-homogeneous polynomial of bi-degree $(2,2)$. Rohn's paper contains two ways to obtain from $\Gamma$ a ruled quartic surface, namely:

(1) Identify the two factors of $\mathbb{P}^{1} \times \mathbb{P}^{1}$ with two skew lines $L_{1}$ and $L_{2}$ in $\mathbb{P}^{3}$. Define $S$ to be the union of the lines through the points $a_{1} \in L_{1}, a_{2} \in L_{2}$ such that $\left(a_{1}, a_{2}\right) \in \Gamma$. Models XIII, no. 1, 2, 3, 4. For these models the singular locus of $S$ is $L_{1} \cup L_{2}$ with multiplicities 2 .

(2) Identify the two factors of $\mathbb{P}^{1} \times \mathbb{P}^{1}$ with a line $L_{1}$ and a conic $C_{2}$ in $\mathbb{P}^{3}$ such that $L_{1}$ does not lie in the plane of $C_{2}$ and $L_{1} \cap C_{2}$ is one point. Define $S$ to be the union of the lines through the points $a_{1} \in L_{1}, a_{2} \in C_{2}$ such that $\left(a_{1}, a_{2}\right) \in \Gamma$ (for special $\Gamma$ ). Model XIII, no. 8. The singular locus of $S$ is $L_{1} \cup C_{2}$ with multiplicities 2.

By a deformation of (1), where $L_{1}$ and $L_{2}$ are moving to a line $L$, Rohn obtains Model XIII, no. 5. The singular locus of $S$ is $L$ with multiplicity 2 .

If $S$ contains a singular line $L$ with multiplicity 3 , then, by an algebraic computation, one obtains explicit equations for $S$. Models XIII no. 6, 7 .

If $S$ contains as singular locus the twisted cubic space curve, then, by an algebraic computation, one finds equations for $S$. Models XIII no. 9, 10.

Now we provide some geometric interpretation of the models.

Case (1) is very explicit. Consider the rational map $M: \mathbb{P}^{3} \cdots \rightarrow \mathbb{P}^{1} \times \mathbb{P}^{1}$, given by $\left(t_{1}, t_{2}, t_{3}, t_{4}\right) \mapsto\left(\left(t_{1}, t_{2}\right),\left(t_{3}, t_{4}\right)\right)$, which is defined ouside the two lines $L_{1}=\{(0,0, *, *)\}$ and $L_{2}=\{(*, *, 0,0)\}$. The fibres of $M$ are lines and $S$ is the Zariski closure of $M^{-1}(\Gamma)$. If $f$ is the bi-homogeneous equation for $\Gamma$ of degree $(2,2)$, then $S$ is defined by $f$ as homogeneous equation of degree 4.

Case (2) is similar but less obvious (probably implicitly in Rohn). The line $L_{1}$ and the conic $C_{2}$ are defined by $t_{3}=t_{4}=0$ and $t_{1}=t_{2} t_{3}-t_{4}^{2}=0$. The rational map $M: \mathbb{P}^{3} \cdots \rightarrow L_{1} \times C_{2}$, given by

$$
\left(t_{1}, t_{2}, t_{3}, t_{4}\right) \mapsto\left(\left(t_{1} t_{3}, t_{2} t_{3}-t_{4}^{2}, 0,0\right),\left(0, t_{4}^{2}, t_{3}^{2}, t_{3} t_{4}\right)\right),
$$

is not defined on $L_{1}$ and $C_{2}$. Almost all fibres of $M$ are lines and the surface $S$ is the Zariski closure of $M^{-1}(\Gamma)$ for a special $\Gamma \subset \mathbb{P}^{1} \times \mathbb{P}^{1}$ and a suitable identification $\mathbb{P}^{1} \times \mathbb{P}^{1}$ with $L_{1} \times C_{2}$.

The geometry of some cases where the surface $S$ has a singular line with multiplicity 3 , is similar to case (1). Using $M: \mathbb{P}^{3} \cdots \rightarrow \mathbb{P}^{1} \times \mathbb{P}^{1}$ as in case (1), the surface $S$ is the Zariski closure of $M^{-1}(\Gamma)$ where $\Gamma \subset \mathbb{P}^{1} \times \mathbb{P}^{1}$ is a curve of bi-degree $(1,3)$.

Also for the case where the twisted cubic curve is singular on $S$ there is a geometric construction for $S$ (implicitly in Rohn). The rational map $M: \mathbb{P}^{3} \cdots \rightarrow \mathbb{P}^{2}$, given by the formula

$$
\left(t_{1}, t_{2}, t_{3}, t_{4}\right) \mapsto(X, Y, Z) \text { with } X=t_{1} t_{3}-t_{2}^{2}, Y=t_{2} t_{3}-t_{1} t_{4}, Z=t_{2} t_{4}-t_{3}^{2},
$$

is defined outside the twisted cubic curve $\left\{\left(s^{3}, s^{2} t, s t^{2}, t^{3}\right) \mid(s, t) \in \mathbb{P}^{1}\right\}$. The closure of the fibres of $M$ are the lines that meet the twisted cubic with total multiplicity 2 . One considers an irreducible conic $\Gamma$ in $\mathbb{P}^{2}$ given by a homogeneous equation $H$. The closure of the $M^{-1}(\Gamma)$ is the ruled quartic surface $S$ with equation $H(X, Y, Z)=0$. Its singular locus is the twisted 
cubic curve. The surface $S$ corresponding to $H=Y^{2}-X Z$ consists of the tangent lines of the twisted cubic curve. There are many more distinct cases for $H$ (see $\S 3.2 .4$ ).

An important contribution to the classification problem is Rohn's theorem stating that, at least in the complex case, the coordinates of the two factors $\mathbb{P}^{1}$ can be chosen such that the equation for a $(2,2)$-curve in $\mathbb{P}^{1} \times \mathbb{P}^{1}$ is symmetric. For the classification over $\mathbb{R}$, which is of course essential for the string models, the situation is more complicated. Rohn has to introduce 'anti-symmetric forms' as well. For case (1) he also dealt with the situation where the two lines $L_{1}, L_{2}$ are complex conjugated. As a consequence case (1) leads to the various models XIII no. 1, 2, 3, 4. In Sect. 4 a 'modern' proof of Rohn's theorem is presented.

About the actual construction of the models we have no information. An inspection of the models shows a metal frame, more or less a cube. On its lower and upper sides quartic curves, namely the intersections with $S$, are present. The strings connect the corresponding points of these two curves. The singular locus of the surface (line(s), conic, cubic space curve) is visible in most models. The symmetry of the equation for $\Gamma$ has disappeared. The geometric interpretation of the family of lines on the surface is evident for only some models.

\subsection{The classification by Cayley and Cremona}

Cremona [5] classified the ruled quartic surfaces in 12 types. We present his statements and results, together with, in brackets [ ], some comments. Let $S \subset \mathbb{P}^{3}$ be a ruled quartic surface, not a cone. $S$ is seen as a 1-parameter family of lines. Through a general point of $S$ there is only one line of the family [This non trivial fact, compare Lemma 1.2, is tacitly assumed by Cremona].

The locus $D$ of the points on $S$ through which there are at least two lines in the 1-parameter family is a curve, called the 'double curve', and has 'in general' degree 3 [ $D$ need not coincide with the singular locus of $S$. Further $D$ can also have degree 2 and even $D=\emptyset$ occurs].

Two intersecting lines of the family determine a plane. The collection $\check{D}$ of all these planes is called the 'bitangent developable'. This 1-dimensional family [assuming $D \neq \emptyset$ ] can be seen as a curve in the dual projective space.

The genus of $S$ is defined as the genus of the (irreducible, singular) curve $H \cap S$ of degree 4, where $H$ is a general plane. Cremona states that the genus can only be 0 or 1 [Missing is the non trivial argument showing that genus 2 is impossible, see Observation 1.4 and [20, Proposition 2.6])]. Then $S$ is classified according to the genus and the nature, i.e., the degrees and multiplicities of the irreducible components, of the curves $D$ and $\check{D}$. In one special case, namely Cremona 9 and Cremona 10 (see below), a relation between $D$ and $\check{D}$ plays a role. The list of the possibilities is obtained by the following construction:

Consider a tuple $\left(C_{1}, C_{2}, f\right)$ consisting of two conics $C_{1}, C_{2} \subset \mathbb{P}^{3}$, not in the same plane, and an isomorphism $f: C_{1} \rightarrow C_{2}$. This defines a ruled surface $S$ which is the union of the lines through the pairs of points $\left\{c_{1}, f\left(c_{1}\right)\right\}$ with $c_{1} \in C_{1}$. In the general case, the line $H_{1} \cap H_{2}$, where $C_{i}$ lies in the plane $H_{i}$ for $i=1,2$, intersects $C_{1}$ in two points $p_{1} \neq q_{1}$ and intersects $C_{2}$ in two points $p_{2} \neq q_{2}$. Now $H_{2} \cap S$ is the union of the conic $C_{2}$ and the two lines through the pairs of points $\left(p_{1}, f\left(p_{1}\right)\right)$ and $\left(q_{1}, f\left(q_{1}\right)\right)$. Thus $S$ is an irreducible ruled surface of degree 4. Moreover, the two lines intersect in a point of the 'double curve' and $H_{2}$ is a 'bitangent plane', i.e., a point on the 'bitangent developable.' The same holds of course for $H_{1}$. Cremona's examples are obtained by varying and degenerating $C_{1}, C_{2}, f$ and he asserts to have found all types in this way [This is not correct since some ruled quartic surfaces are only obtained from a line and a curve of degree 3 . However, by including 'reciprocal 
surfaces' and maybe stretching the meaning of 'degeneration', some other surfaces can be obtained].

Before giving Cremona's list we need to explain to notion of 'reciprocal surface' or 'dual surface' in more modern terms, of a surface $S \subset \mathbb{P}^{3}$. It is obtained by considering all tangent planes at the nonsingular points of $S$. Each tangent plane is a point in the dual projective space $\check{\mathbb{P}^{3}}$. The Zariski closure of all these points is the dual surface $\check{S} \subset \check{\mathbb{P}^{3}}$. In the case that $S$ is ruled, also $\breve{S}$ is ruled and has the same degree as $S$. In the special case that $S$ is a ruled quartic surface, the dual $\check{S}$ is again a ruled quartic surface and the 'double line' of $\check{S}$ can be seen to be the 'bitangent developable' of $S$. Cremona's list shows that the species 3 and 4 are dual and similar for the species 7 and 8 . The other types are 'selfdual'.

In the table one has to give 'double curve' the interpretation 'singular locus'. The genus $g$ of the surface is 0 , except for Cremona 11, 12 where it is 1 . We adopt a notation of Cayley, namely the expression $d^{m}$ stands for an irreducible component of the singular locus of degree $d$ and with multiplicity $m$. The difference between Cremona 6 and Cremona 11 is that the two lines intersect in the first case and are skew in the second one. The difference between Cremona 9 and Cremona 10 is somewhat subtle. In case 10 the bitangent planes are the planes containing the singular line, denoted by 1 . In case 9 , the bitangent planes are the planes containing another line, denoted by $1^{\prime}$.

\begin{tabular}{llllll}
\hline Double curve & $\begin{array}{l}\text { Double curve } \\
\text { recip. surface }\end{array}$ & Cremona type & Double curve & $\begin{array}{l}\text { Double curve } \\
\text { recip. surface }\end{array}$ & Cremona type \\
\hline $3^{2}$ & $3^{2}$ & 1 & $3^{2}$ & $1^{3}$ & 7 \\
$2^{2}, 1^{2}$ & $2^{2}, 1^{2}$ & 2 & $1^{3}$ & $3^{2}$ & 8 \\
$1^{3}$ & $2^{2}, 1^{2}$ & 3 & $1^{3}$ & $1^{3}$ & 9 \\
$2^{2}, 1^{2}$ & $1^{3}$ & 4 & $1^{3}$ & $1^{3}$ & 10 \\
$1^{2}, 1^{2}, 1^{2}$ & $1^{2}, 1^{2}, 1^{2}$ & 5 & $1^{2}, 1^{2}$ & $1^{2}, 1^{2}$ & $11, g=1$ \\
$1^{2}, 1^{2}$ int & $1^{2}, 1^{2}$ int & 6 & $1^{2}$ & $1^{2}$ & $12, g=1$ \\
\hline
\end{tabular}

The approach of Cayley [3] (and of Rohn) has the classical name "analytic geometry", indicating the use of coordinates and algebraic operations with formulas. In contrast, Cremona's (and Sturm's) approach is purely "synthetic". As a consequence, Cremona's paper is difficult to read and his results are not easily verified.

Cayley's method consists of taking three curves in $\mathbb{P}^{3}$ and to consider the ruled surface $S$ which is the union of the lines meeting the three curves. Using a formula for the degree of $S$, he now computes possibilities of ruled quartic surfaces. The expression "the six coordinates of a line" in Cayley's work indicates that the Grassmann variety $\operatorname{Gr}(2,4)$ of the lines in $\mathbb{P}^{3}$ plays a role. Moreover, Cayley made explicit calculations of reciprocal surfaces.

Cremona, in competition with Cayley it seems, gives in [5] an inadequate account of Cayley's work on ruled quartic surfaces. The classification by Cremona has been accepted by later authors, e.g., in the book of W.L. Edge [7].

There are however some critical comments by R. Sturm. In Rohn's paper [12], p.147, there is an explicit equation

$$
w x^{2}(x+3 N z)+F_{4}(x, y)=0, F_{4} \text { a binary quartic, } N \text { a constant }
$$

(in homogeneous coordinates $x, y, z, w)$ and the remark in a footnote: "this ruled surface is not mentioned by Cremona in his treatise". Unfortunately, the equation is wrong. It does not define a ruled surface. But Rohn's geometric construction is valid and is indeed not explicitly mentioned by Cremona. It could be interpreted as hiding in Cremona's species 10. 
In Pascal's well written Repertorium, the classification by Cayley and Cremona is reviewed, [11], XII, §10, and there Rohn's extra case reappears on p. 338-339 with the same correct geometric construction and another wrong formula. In the classification of the present paper Rohn's example is Number 5. Bottema [2, p. 349] mentions Rohn's extra case and points out that Cremona's treatment of the case where the double curve is $3^{2}$, is unsatisfactory.

We mention, as a curiosity, the thesis of Wong [23]. There, a rational morphism $\mathbb{P}^{3} \cdots \rightarrow$ $\operatorname{Gr}(2,4)$, associated to the classical 'tetrahedral complex', is considered. Certain plane curves in $\mathbb{P}^{3}$ of degree 2 and 3 have as images in $\operatorname{Gr}(2,4)$ curves of degree 4 and correspond therefore to ruled quartic surfaces. He claims that every 'species' in Cremona's list is obtained in this way.

For other details on the early history of the subject we refer to the contribution of W.Fr. Meyer in [9] and the References of the present paper. These historical facts and the critical remarks are our motivation to investigate the classification of ruled quartic surfaces.

\subsection{A modern classification}

We indicate the notions and background needed for the proposed modern classification and for the understanding of the table containing the classification. In later sections details and proofs are presented.

A ruled quartic surface $S$ corresponds to an irreducible curve $C$ on the Grassmann variety $\operatorname{Gr}(2,4)$ of the lines in $\mathbb{P}^{3}$. This Grassmann variety is in a natural way a non singular quadric in $\mathbb{P}^{5}$ and $C$ has degree 4 w.r.t. this embedding. Let $C^{\text {norm }} \rightarrow C$ denote the normalization of $C$. The genus of $C^{\text {norm }}$ is 0 or 1 and $C^{\text {norm }}=C$ if its genus is 1 .

The curve $C$ can have a singularity, in fact only a node or a cusp. Let $P(C) \subset \mathbb{P}^{5}$ denote the smallest projective subspace containing $C \subset \operatorname{Gr}(2,4) \subset \mathbb{P}^{5}$. The dimension of $P(C)$ can be 3 or 4 . The projective subspace $P(C)$ of $\mathbb{P}^{5}$ can lie on 0,1 or 2 tangent spaces of $\operatorname{Gr}(2,4)$.

The tautological bundle on $\operatorname{Gr}(2,4)$, restricted to $C$ and pulled back to $C^{\text {norm }}$ is a vector bundle $B$ of rank 2 on $C^{\text {norm }}$. If $C^{\text {norm }} \cong \mathbb{P}^{1}$, then there are two possibilities for $B$, namely $O_{\mathbb{P}^{1}}(-1) \oplus O_{\mathbb{P}^{1}}(-3)$ and $O_{\mathbb{P}^{1}}(-2) \oplus O_{\mathbb{P}^{1}}(-2)$.

In some situations, a morphism $f: \mathbb{P}^{1} \rightarrow \mathbb{P}^{1}$ of degree 3 is involved. The three possibilities for $f$ (assuming characteristic 0 ) are indicated by $a, b, c$.

An item of the table of the classification is given by the above discrete invariants, the structure of the singular locus and sometimes an additional discrete invariant. Every case corresponds to an irreducible moduli space of ruled quartic surfaces, implicit in the calculations. We have not found a natural numbering of the 29 cases. The item 'Number' appearing in the tables is introduced for notational reasons and refers to the computations in $\S 3.1$.

The cases with $C^{\text {norm }}$ of genus 0 and $B$ of type $-1,-3$ are:

\begin{tabular}{|c|c|c|c|c|c|c|}
\hline Number & $\begin{array}{l}\text { singular } \\
\text { locus }\end{array}$ & $\operatorname{dim} P(C)$ & $\begin{array}{l}\text { singularities } \\
\text { of } C\end{array}$ & tangent spaces & Cremona & XIII \\
\hline $1 a, b, c$ & $1^{3}$ & 3 & - & 2 & 9 & \\
\hline $2 a, b, c$ & $3^{2}$ & 4 & - & 1 & 7 & \\
\hline $3 a, b$ & $1^{2}, 2^{2}$ & 4 & - & 1 & 4 & \\
\hline 4 & $1^{3}$ & 3 & node & 1 & 10 & 7 \\
\hline 5 & $1^{3}$ & 3 & cusp & 1 & 10 & \\
\hline
\end{tabular}


The cases with $C^{\text {norm }}$ of genus 0 and $B$ of type $-2,-2$ are:

\begin{tabular}{lllllll}
\hline Number & singular locus & $\operatorname{dim} P(C)$ & $\begin{array}{l}\text { singularities } \\
\text { of } C\end{array}$ & tangent spaces & Cremona & XIII \\
\hline 6 & $1^{2}, 1^{2}, 1^{2}$ & 3 & node & 2 & 5 \\
7 & $1^{2}, 1^{2}, 1^{2}$ & 3 & cusp & 2 & 5 & \\
8 & $1^{3}$ & 4 & - & 1 & 3 & 3 \\
9 & $1^{3}$ & 4 & - & 1 & 6 & \\
$10 \mathrm{a}$ & $1^{2}, 1^{2}$, int & 3 & node & 1 & 6 & \\
$10 \mathrm{~b}$ & $1^{2}, 1^{2}$, int & 3 & cusp & 1 & 2 & 8 \\
11 & $1^{2}, 2^{2}$ & 4 & - & 1 & 2 & 6 \\
12 & $1^{2}, 2^{2}$ & 4 & - & 1 & 8 & 9,10 \\
13 a,b,c & $1^{3}$ & 4 & - & 1 & 1 & \\
$14 \ldots$ & $3^{2}$ & 4 & - & - & & \\
\hline
\end{tabular}

It is shown in $\$ 3.2 .4$ that Number 14 consists of six distinct cases. In what discrete aspect Number 11 and 12 differ is explained in $\$ 3.1$. The reciprocals of $2 a, b, c$ are $13 a, b, c$. The reciprocals of $3 a, b$ are 8,9 (and thus showing that Number 8 and 9 are distinct). The other examples are 'selfdual'.

The cases with $C^{\text {norm }}=C$ of genus 1 are:

\begin{tabular}{llllll}
\hline Number & singular locus & $\operatorname{dim} P(C)$ & tangent spaces & Cremona & XIII \\
\hline 15 & $1^{2}$ & 3 & 1 & 12 & 5 \\
16 & $1^{2}, 1^{2}$ & 3 & 2 & 11 & $1,2,3,4$ \\
\hline
\end{tabular}

Now we summarize the remaining part of this paper.

In Sect. 1, well known, useful properties of the Grassmann variety $\operatorname{Gr}(2,4)$ are collected. The relation between curves on $\operatorname{Gr}(2,4)$ and ruled surfaces in $\mathbb{P}^{3}$ is studied in detail. The vector bundle $B$ on $C^{\text {norm }}$ is introduced. It is shown how $B$ can be used to obtain an equation for a ruled surface $S \subset \mathbb{P}^{3}$ and the structure of the normalisation of $S$ (the latter is a ruled surface in the modern sense).

A list of possibilities for the singular locus of $S$ is deduced from the possibilities for the singularities of a general hyperplane section of $S$ which is an irreducible plane quartic of genus 0 or 1 . The useful notion 'reciprocal of a ruled surface' is given in 'modern' terms.

The above material is used in Sect. 2 to obtain the well known classification of ruled cubic surfaces. $\S 3.1$ is a case by case study of ruled quartic surfaces. For the most complicated situation, where the genus of $C^{\text {norm }}$ is 0 , one considers two meromorphic sections $a, b$ of the vector bundle $B$, giving rise to morphisms $\bar{a}, \bar{b}: \mathbb{P}^{1} \rightarrow \mathbb{P}^{3}$. The surface $S$ consists of the lines connecting the pairs of points $\{\bar{a}(p), \bar{b}(p)\}$ with $p \in$ $\mathbb{P}^{1}$.

By considering linear combinations of $a, b$ and by choosing homogeneous coordinates for $\mathbb{P}^{1}$ and $\mathbb{P}^{3}$, one obtains 'normal forms' and 'normal' equations for $S$. Details are given in some cases and in many cases only the final result is presented. The limitation of this process is compensated by a second method in $\S 3.2$, namely deriving equations for $S$ using a given singular locus. Moreover, $\S 3.2$ is used as a verification of the results of $\S 3.1$.

Section 4 provides a proof of Rohn's forgotten remarkable theorem using real and complex elliptic curves and their degenerations. 


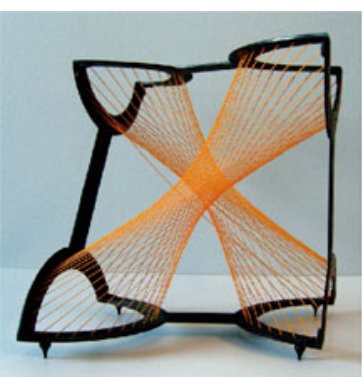

XIII 4

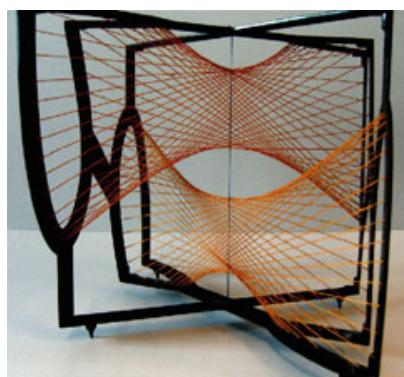

XIII 5

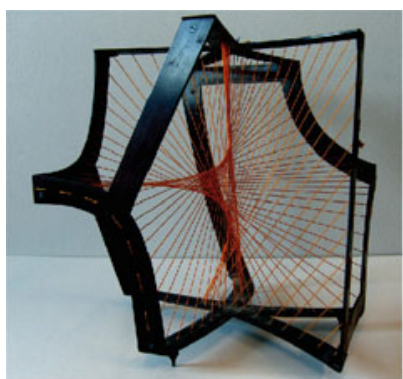

XIII 7

\section{Curves on the Grassmann variety $\operatorname{Gr}(2,4)$}

\subsection{Properties of the Grassmann variety}

Let $V$ be a vector space of dimension 4 over the (algebraically closed) field $K$. The lines in the projective space $\mathbb{P}(V) \cong \mathbb{P}^{3}$ are points of the Grassmann variety $G r:=\operatorname{Gr}(2, V)=\operatorname{Gr}(2,4)$ and the natural way to study a ruled surface $S \subset \mathbb{P}(V)$ is to consider the set of the lines on $S$ as subset of $G r$. We briefly define $G r$ and summarize its main properties.

For notational convenience we fix a basis $e_{1}, e_{2}, e_{3}, e_{4}$ of $V$ and we identify the exterior power $\Lambda^{4} V$ with $K$ by $e_{1} \wedge \cdots \wedge e_{4} \mapsto 1$. The obvious symmetric bilinear map $\Lambda^{2} V \times \Lambda^{2} V \rightarrow$ $\Lambda^{4} V=K$ is non degenerate.

A line in $\mathbb{P}(V)$ correspond to a plane $W \subset V$, a line $\Lambda^{2} W \subset \Lambda^{2} V$ and to a point in $\mathbb{P}\left(\Lambda^{2} V\right) \cong \mathbb{P}^{5}$. If $W$ has basis $v_{1}, v_{2}$, then $w=v_{1} \wedge v_{2}$ is a basis vector for $\Lambda^{2} W$ and $\bar{w}:=K w$ is this point of $\mathbb{P}\left(\Lambda^{2} V\right)$.

$G r=G r(2, V) \subset \mathbb{P}\left(\Lambda^{2} V\right)$ consists of all these points. Now $\bar{w}$ (with $w \in \Lambda^{2} V, w \neq 0$ ) belongs to $G r$ if and only if $w$ is decomposable, i.e., has the form $v_{1} \wedge v_{2}$. The latter is equivalent to $w \wedge w=0$. We use the six elements $e_{i j}:=e_{i} \wedge e_{j}, i<j$ as basis for $\Lambda^{2} V$ and write an element of this vector space as $\sum_{i<j} p_{i j} e_{i j}$. The $p_{i j}$ are called the Plücker coordinates. They also serve as homogeneous coordinates for $\mathbb{P}\left(\Lambda^{2} V\right)$. One finds that $G r$ is the non degenerate quadric given by the equation $p_{12} p_{34}-p_{13} p_{24}+p_{14} p_{23}=0$. For notational purposes and for convenience of the reader we recall the following.

List of properties of $\mathrm{Gr}$ (of importance for our purposes).

(i) $p_{0}, \ell_{0}, h_{0}$ are a point, a line and a plane of $\mathbb{P}(V)=\mathbb{P}^{3}$. One identifies $p_{0}$ with a $\bar{v}_{0}, v_{0} \in V, v_{0} \neq 0$ and $\ell_{0}$ with a $\bar{w}_{0}, w_{0} \in \Lambda^{2} V, w_{0} \wedge w_{0}=0$. We note that $\bar{w} \in \mathbb{P}\left(\Lambda^{2} V\right)$ with $w \wedge w=0$ is both seen as a point of $G r$ and as a line in $\mathbb{P}(V)$.

(ii) Two lines $\bar{w}_{1}, \bar{w}_{2}$ of $\mathbb{P}(V)$ intersect if and only if $w_{1} \wedge w_{2}=0$.

(iii) Every hyperplane of $\mathbb{P}\left(\Lambda^{2} V\right)$ has the form $\{\bar{z} \mid w \wedge z=0\}$ with $w \in \Lambda^{2} V, w \neq 0$ and unique $\bar{w}$. If $w$ is indecomposable, then the intersection of the hyperplane with $G r$ is a non degenerate quadric.

If $w$ is decomposable, i.e., $\bar{w}=\bar{w}_{0} \in G r$, corresponding to the line $\ell_{0}$, then the hyperplane is the tangent plane $T_{G r, \bar{w}_{0}}$ of $G r$ at $\bar{w}_{0}$. The intersection $T_{G r, \bar{w}_{0}} \cap G r$ is singular and can be identified with the cone in $\mathbb{P}^{4}$ over a nonsingular quadric in $\mathbb{P}^{3}$. This intersection identifies with $\sigma_{1}\left(\ell_{0}\right):=$ the collection of all lines $\ell$ with $\ell \cap \ell_{0} \neq$ $\emptyset$. Consider for example $w_{0}=e_{1} \wedge e_{2}$. This intersection is now $\left\{\left\{p_{i j}\right\} \mid p_{34}=\right.$ $\left.0,-p_{13} p_{24}+p_{14} p_{23}=0\right\}$. The vertex $\bar{w}_{0}$ of this cone is its only singular point. 
(iv) $\sigma_{2}\left(p_{0}\right):=$ the collection of all lines through $p_{0}$; this is a 2-plane in $G r$. Indeed, take $p_{0}=\bar{e}_{1}$. Then $\sigma_{2}\left(p_{0}\right)=\left\{\overline{\sum_{1<j \leq 4} p_{1 j} e_{1} \wedge e_{j}} \mid\right.$ no relations $\}$ (called a $\omega$-plane in [7]).

(v) $\sigma_{1,1}\left(h_{0}\right):=$ the collection of all lines in the plane $h_{0}$. This is a 2-plane in $G r$. Indeed, take $h_{0}=\left\langle e_{1}, e_{2}, e_{3}\right\rangle$. Then this collection identifies with $\left\{\overline{\sum_{1 \leq i<j \leq 3} p_{i j} e_{i} \wedge e_{j}} \mid\right.$ no relations $\}$ (called a $\rho$-plane in [7]).

(vi) $\sigma_{2,1}\left(p_{0}, h_{0}\right):=$ is the collection of all lines in $h_{0}$ through $p_{0}$. This is a line on $G r$. Indeed, take $h_{0}=\overline{\left\langle e_{1}, e_{2}, e_{3}\right\rangle}, p_{0}=\bar{e}_{1}$. Then this collection identifies with $\left\{\overline{\sum_{j=2,3} p_{1 j} e_{1} \wedge e_{j}} \mid\right.$ no relations $\}$.

(vii) Every plane in $G r$ has the form $\sigma_{2}\left(p_{0}\right)$ or $\sigma_{1,1}\left(h_{0}\right)$. Every line in $G r$ has the form $\sigma_{2,1}\left(p_{0}, h_{0}\right)$ and is thus the intersection of a (uniquely determined) pair of 2-planes in $G r$ of different type.

(viii) There are three types of projective subspaces $P \subset \mathbb{P}\left(\Lambda^{2} V\right)$ of dimension 3 with respect to their relation with $G r$, namely:

(a) $G r \cap P$ is a non degenerate quartic surface. The equations of $P$ are $p_{12}=p_{34}=0$ for a suitable basis of $V$. Moreover $P$ lies in precisely two tangent space, namely $T_{G r, \overline{e_{12}}}$ and $T_{G r}, \overline{e_{34}}$.

(b) $G r \cap P$ is an irreducible degenerate quartic surface. The equations of $P$ are $p_{34}=$ $p_{13}+p_{24}=0$ for a suitable basis of $V$. Now $P$ lies on only one tangent space, namely $T_{G r, \overline{e_{12}}}$ and $G r \cap P$ is the cone $p_{13}^{2}+p_{14} p_{23}=p_{34}=p_{13}+p_{24}=0$ over the quadric curve $p_{13}^{2}+p_{14} p_{23}=0$.

(c) $G r \cap P$ is reducible. The equations for $P$ are $p_{12}=p_{13}=0$ for a suitable basis of $V$. Further, $G r \cap P$ is the union of the planes $p_{14}=0$ and $p_{23}=0$.

\subsection{Ruled surfaces and curves on $\mathrm{Gr}$}

Lemma 1.1 (1) Let $C \subset G r$ be an irreducible curve of degree $d \geq 2$, not lying in some 2-plane $\sigma_{2}\left(p_{0}\right)$. Then $\tilde{S}:=\{(\bar{w}, \bar{v}) \in C \times \mathbb{P}(V) \mid w \wedge v=0\}$ is an irreducible variety of dimension 2. Its image $S$ under the projection map $\mathrm{pr}_{2}: \tilde{S} \rightarrow \mathbb{P}(V)$ is an irreducible surface of degree e. Suppose that through a general point of $S$ there are $f$ lines $\bar{w} \in C$. Then $d=e \cdot f$.

(2) Let $P(C)$ denote the smallest projective subspace of $\mathbb{P}\left(\Lambda^{2} V\right)$, containing $C$. If $d \geq 3$ and $S$ is not a cone, a plane or a quadric, then $\operatorname{dim} P(C) \geq 3$.

Proof (1) We note that $C \subset \sigma_{2}\left(p_{0}\right)$ is not interesting since then $S$ is a cone. The fibres of $p r_{1}: \tilde{S} \rightarrow C$ are lines in $\mathbb{P}(V)$ and the fibres of $p r_{2}: \tilde{S} \rightarrow S$ are finite. Thus $S$ is an irreducible ruled surface of some degree $e$. A general line $\bar{w}_{0}$ in $\mathbb{P}(V)$ intersects $S$ in $e$ points. Through each of these $e$ points there are $f$ lines $\bar{w} \in C$. Thus the intersection of $C$ with the general hyperplane $\left\{\bar{w} \in \mathbb{P}\left(\Lambda^{2} V\right) \mid w \wedge w_{0}=0\right\}$ consists of $e \cdot f$ points and therefore $d=e \cdot f$.

(2) Since $d>1$, one has $\operatorname{dim} P(C)>1$. Suppose that $\operatorname{dim} P(C)=2$. If $P(C) \subset G r$, then either $P(C)$ is a $\sigma_{2}\left(p_{0}\right)$ and $S$ is a cone, or $P(C)$ is a $\sigma_{1,1}\left(h_{0}\right)$ and $S$ is the plane $h_{0}$. If $P(C) \not \subset G r$, then $C \subset P(C) \cap G r$ is a curve of degree at most 2 and $S$ is a plane or a quadric. Hence $\operatorname{dim} P(C) \geq 3$.

In the sequel we consider ruled surfaces (reduced, irreducible) $S \subset \mathbb{P}(V)$ of some degree $d \geq 3$ which are not cones. One associates to $S$ the subset $\tilde{C}$ of $G r$ corresponding to the lines on $S$. 
Lemma 1.2 $\tilde{C}$ is the union of an irreducible curve $C$ (not lying in some 2-plane $\sigma_{2}\left(p_{0}\right)$ ) of degree $d$ and a finite, possibly empty, set. Moreover, through a general point of $S$ there is one line of the surface.

Proof Consider the affine open part of $G r$ given by $p_{12} \neq 0$. The points of this affine part, actually $\cong \mathbb{A}^{4}$, can uniquely be written as planes in $V$ with basis $e_{1}+a e_{3}+b e_{4}, e_{2}+c e_{3}+d e_{4}$ and correspond to the vectors

$$
e_{12}+c e_{13}+d e_{14}+a e_{23}+b e_{24}+(a d-b c) e_{34} .
$$

Let $F\left(t_{1}, \ldots, t_{4}\right)=0$ be the homogeneous equation of $S$. The intersection of $\tilde{C}$ with this affine part consists of the tuples $(a, b, c, d)$ such that $F(s, t, a s+c t, b s+d t)=0$ for all $(s, t) \neq(0,0)$. Write this expression as a homogeneous form in $s, t$ and coefficients polynomials in $a, b, c, d$. Then the ideal generated by these polynomials in $a, b, c, d$ defines the intersection of $\tilde{C}$ with this affine part of $G r$. Thus $\tilde{C}$ is Zariski closed.

Clearly $\tilde{C}$ has dimension 1 and can be written as the union of irreducible curves $C_{i}, i=$ $1, \ldots, r$ and a finite set. The image of the projection $\left\{(\bar{w}, \bar{v}) \in C_{1} \times \mathbb{P}(V) \mid w \wedge v=0\right\} \rightarrow \mathbb{P}(V)$ is a ruled surface contained in $S$. Since $S$ is irreducible, the image is $S$. If $r \geq 2$, then, through a point $\bar{v}$ of a line $\bar{w}_{2} \in C_{2}, \bar{w}_{2} \notin C_{1}$ passes a line $\bar{w}_{1} \in C_{1}$. Hence $w_{1} \wedge w_{2}=0$ for all $\overline{w_{1}} \in C_{1}$ and thus $w \wedge w_{2}=0$ for all $\bar{w} \in P\left(C_{1}\right)$. By symmetry $w_{1} \wedge w_{2}=0$ for all $\bar{w}_{1} \in P\left(C_{1}\right), \bar{w}_{2} \in P\left(C_{2}\right)$. Since the symmetric bilinear form $\left(w_{1}, w_{2}\right) \mapsto w_{1} \wedge w_{2}$ on $\Lambda^{2} V$ is not degenerate, one obtains a contradiction by comparing dimensions: $\operatorname{dim} P\left(C_{1}\right) \geq$ 3, $\operatorname{dim} P\left(C_{2}\right) \geq 3, \operatorname{dim} \mathbb{P}\left(\Lambda^{2} V\right)=5$. We conclude that the $f$ of Lemma 1.1 is 1 and that the degree of $C$ is $d$.

Lemma 1.3 Let $\bar{w}_{0} \in \tilde{C} \backslash C$, then $C$ lies in the tangent space of $G r$ at $\bar{w}_{0}$. In other words, the line $\bar{w}_{0}$ intersects every line on $S$, belonging to $C$.

Proof If the tangent space at $\bar{w}_{0}$ does not contain $C$, then the intersection $C \cap T_{G r}, \bar{w}_{0}$ consists of $d$ points, counted with multiplicity. Thus the line $\bar{w}_{0}$ on $S$ intersects $d$ lines of $S$, corresponding to points of $C$. Let $H \subset \mathbb{P}(V)$ be a plane through $\bar{w}_{0}$. The intersection $H \cap S$ consists of $\bar{w}_{0}$ and a curve $\Gamma$ of degree $d-1$. Therefore $\Gamma \cap \bar{w}_{0}$ consists of $d-1$ points (counted with multiplicity), instead of the $d$ points that we expect. This contradiction proves the lemma.

Definition 1.4 The lines on $S$ corresponding to the points of $\tilde{C} \backslash C$ will be called here isolated lines. A line $\bar{w}_{1}$ on $S$ is, classically, called a directrix if $\bar{w}_{1}$ meets every line $\bar{w}_{2}$ with $\bar{w}_{2} \in C$. Thus an isolated line is a directrix. It is also possible that a line $\bar{w}_{1} \in C$ is a directrix. The classical concept of 'double curve' on $S$ is, according to [7], p. 8, (the Zariski closure of) the set of points on $S$ lying on at least two, non isolated, lines of $S$.

Observation 1.5 Let $C \subset P=\mathbb{P}^{r}, r \geq 3$ be an irreducible curve of degree 4 and such that $C$ does not lie in a proper subspace of $P$. Let $g \leq 2$ be the genus of the normalization $n: C^{\text {norm }} \rightarrow C$. Then one of the following holds:

(1) $g=0, r=4, C$ is the, non singular, rational normal quartic.

(2) $g=0, r=3, C$ is non singular or has one singular point which is a node or ordinary cusp.

(3) $g=1, r=3$ and $C$ is non singular.

Moreover, if $C$ lies on a quadratic cone in $\mathbb{P}^{3}$, then $g=1$ or $g=0$ and $C$ has a singular point. 
Proof Let $I$ be the sheaf of ideals of $C \subset P$. The exact sequence

$$
0 \rightarrow I \otimes O_{P}(1) \rightarrow O_{P}(1) \rightarrow \mathcal{L} \rightarrow 0
$$

with $\mathcal{L}=O_{P}(1) \otimes O_{C}$ and the minimality of $r$ implies that $H^{0}\left(O_{P}(1)\right) \rightarrow H^{0}(\mathcal{L})$ is injective and thus $1+r \leq \operatorname{dim} H^{0}(\mathcal{L})$. Define the skyscraper sheaf $\mathcal{Q}$ on $C$ by the exact sequence of sheaves on $C, 0 \rightarrow \mathcal{L} \rightarrow n_{*} n^{*} \mathcal{L} \rightarrow \mathcal{Q} \rightarrow 0$. One finds that

$4 \leq 1+r \leq \operatorname{dim} H^{0}(C, \mathcal{L}) \leq \operatorname{dim} H^{0}\left(C^{\text {norm }}, n^{*} \mathcal{L}\right)=1-g+4+\operatorname{dim} H^{1}\left(C^{\text {norm }}, n^{*} \mathcal{L}\right)$. $H^{1}\left(C^{\text {norm }}, n^{*} \mathcal{L}\right)=0$, since the degree of $n^{*} \mathcal{L}$ is 4 and $g \leq 2$. Thus $g=2$ is not possible. For $g=1$, one has $H^{0}(C, \mathcal{L})=H^{0}\left(C^{\text {norm }}, n^{*} \mathcal{L}\right)$ and $C=C^{\text {norm }}$ since $n^{*} \mathcal{L}$ is very ample on $C^{\text {norm }}$. Let $E$ be an elliptic curve with neutral element $e$, then $H^{0}(E, 4[e])$ has basis $t_{1}=1, t_{2}=x, t_{3}=y, t_{4}=x^{2}$ (in the standard notation) and $E$ lies on the quadratic cone $t_{2}^{2}-t_{1} t_{4}=0$.

For $g=0$, the curves $C \subset P$ are parametrized by polynomials of degree $\leq 4$ in a variable $t$. Hence $r \leq 4$. For $r=4$, the only possibility is $t \mapsto\left(1, t, t^{2}, t^{3}, t^{4}\right)$. For $r=3$, one has the examples:

$t \mapsto\left(1, t, t^{3}, t^{4}\right)$ and $C$ is non singular,

$t \mapsto\left(1, t^{2}, t^{3}, t^{4}\right)$ and $C$ has an ordinary cusp,

$t \mapsto\left(t, t^{2}, t^{3}, t^{4}-1\right)$ and $C$ has a node.

In general, by intersecting $C$ with planes $H \subset \mathbb{P}^{3}$, through one singular point (or more), one can verify that $C$ has at most one singular point and that such a point can only be a node or an ordinary cusp.

Finally, if $g=0$ and $C$ is contained in a quadratic cone in $\mathbb{P}^{3}$, then $C$ is singular (see [8], exercise IV, 6.1). According to the examples, this singular point can be either a node or a cusp. We note that the exercises IV, 3.4, 3.6 and II, Example 7.8.6 of [8] are closely related to the above reasoning.

Corollary 1.6 A ruled surface of degree $d \geq 3$ can have at most two isolated lines. If $S$ has two isolated lines $\bar{w}_{1}, \bar{w}_{2}$, then $\bar{w}_{1} \cap \bar{w}_{2}=\emptyset$.

Proof The first statement follows from $\operatorname{dim} P(C) \geq 3$. If $\bar{w}_{1} \cap \bar{w}_{2} \neq \varnothing$, then $C$ lies in $G r \cap T_{G r}, \bar{w}_{1} \cap T_{G r}, \bar{w}_{2}$. According to the List of properties of $G r$, (viii) part (c), the latter is the union of two planes. One of them contains $C$ and this contradicts $\operatorname{dim} P(C) \geq 3$.

Corollary 1.7 (1) A general line of a 'general' ruled surface $S$ of degree $d \geq 3$ meets $d-2$ other lines of $S$, corresponding to points of $C$. In particular, the 'double curve' is not empty. However:

(2) Let $T C \subset \mathbb{P}^{3}$ be the twisted cubic curve. The equation of the surface $S$ consisting of the tangents of TC is $\left(t_{1} t_{4}-t_{2} t_{3}\right)^{2}-4\left(t_{1} t_{3}-t_{2}^{2}\right)\left(t_{2} t_{4}-t_{3}^{2}\right)=0$.

The singular locus of $S$ is TC and no two distinct lines of the surface intersect.

Proof (1) For a general point $\bar{w}_{0} \in C$, the intersection $C \cap T_{G r}, \bar{w}_{0}$ is a positive divisor on $C$ of degree $d$, with support in the non singular locus of $C$ and $\geq 2\left[\bar{w}_{0}\right]$. For a 'general' $S$ the divisor will be $2\left[\bar{w}_{0}\right]+\sum_{i=1}^{d-2}\left[\bar{w}_{i}\right]$ with distinct points $\bar{w}_{i} \in C, i=0, \ldots, d-2$. Thus $\bar{w}_{0}$ meets precisely $d-2$ other lines corresponding to points of $C$.

(2) Let $t \mapsto\left(1, t, t^{2}, t^{3}\right) \in \mathbb{P}^{3}$ be $T C$ in parametrized form. The tangent line $\bar{w}_{t}$ goes through the point $\left(0,1,2 t, 3 t^{2}\right)$ and has Plücker coordinates

$$
p_{12}=1, p_{13}=2 t, p_{14}=3 t^{2}, p_{23}=t^{2}, p_{24}=2 t^{3}, p_{34}=t^{4} .
$$


This defines the non singular curve $C \subset G r$ corresponding to $S$. From $\bar{w}_{t} \wedge \bar{w}_{s}=$ $(t-s)^{4}$ it follows that the tangent lines do not intersect for $t \neq s$. In other terms $T_{G r, \bar{w}_{0}} \cap C=4\left[\bar{w}_{0}\right]$ for every $\bar{w}_{0} \in C$.

Proposition 1.8 (1) $p r_{2}: \tilde{S}:=\{(\bar{w}, \bar{v}) \in C \times \mathbb{P}(V) \mid w \wedge v=0\} \rightarrow S$ is a birational morphism. Let $C^{\text {norm }} \rightarrow C$ denote the normalization of $C$ and let $\tilde{\tilde{S}}=\tilde{S} \times{ }_{C} C^{\text {norm }}$ be the pullback of $\tilde{S} \rightarrow C$. Then $\tilde{\tilde{S}} \rightarrow C^{\text {norm }}$ is a ruled surface (in the modern sense) and $\tilde{\tilde{S}} \rightarrow S$ is the normalization of $S$.

(2) The singular locus of $S$ is purely 1-dimensional or empty.

(3) Suppose that the line $\bar{w}$ belongs to the singular locus of $S$ and does not correspond to a singular point of $C$. Then $C$ lies in the tangent space of $G r$ at the point $\bar{w}$.

Proof (1) The morphism is finite since $\mathrm{pr}_{2}^{-1}(\bar{v})$ is the finite set of lines of $S$ through $\bar{v} \in S$. For a general $\bar{v} \in S$, this set has one element and therefore the degree of $p r_{2}$ is 1 and so $p r_{2}$ is birational.

The fibres of $p r_{1}: \tilde{S} \rightarrow C$ are isomorphic to $\mathbb{P}^{1}$ and the same holds for the fibres of $\tilde{\tilde{S}} \rightarrow C^{\text {norm }}$. Therefore the latter is a ruled surface in the modern terminology. Moreover the morphism $\tilde{\tilde{S}} \rightarrow \tilde{S}$ is birational and so $\tilde{\tilde{S}} \rightarrow S$ is the normalization.

(2) The local ring of an isolated singular point of $S$ is normal and will remain a singular point of the normalization of $S$. Since $\tilde{\tilde{S}}$ is smooth, $S$ has no isolated singularities.

(3) This follows from Lemma 1.3 since, by assumption, $\bar{w} \in \tilde{C} \backslash C$.

Remark 1.9 The 'double curve', as defined above, is seen, by Proposition 1.8, to be part of the singular locus of $S$. The genus of $S$ is defined as the genus of $\tilde{\tilde{S}}$ and thus is equal to the genus of $C^{\text {norm }}$.

Lemma 1.10 Suppose that $\operatorname{dim} P(C)=3$ and that $P(C)$ is the intersection of two tangent spaces of $G r$ at points $\bar{w}_{1} \neq \bar{w}_{2}$. Then the lines $\bar{w}_{1}, \bar{w}_{2}$ do not intersect. For a suitable choice of the homogeneous coordinates $t_{1}, t_{2}, t_{3}, t_{4}$ of $\mathbb{P}(V)$, the equation $F$ of $S$ is bi-homogeneous of degree $\left(a_{1}, a_{2}\right)$, with $a_{1}+a_{2}=d$, in the pairs $t_{1}, t_{2}$ and $t_{3}, t_{4}$. Further $\tilde{C} \backslash C=\left\{\bar{w}_{1}, \bar{w}_{2}\right\}$.

The lines $\bar{w}_{1}, \bar{w}_{2}$ are 'directrices'. The singular locus of $S$ consists of the lines $\bar{w}_{i}$ with $a_{i}>1$ and for each singular point $\bar{w} \in C$, the line $\bar{w} \subset S$.

Proof The assumption that the lines $\bar{w}_{1}, \bar{w}_{2}$ intersect, yields, according to (viii) part (c), the contradiction that $C$ lies in a plane. Take $w_{1}=e_{12}$ and $w_{2}=e_{34}$, then $P(C)=$ $T_{G r}, \overline{e_{12}} \cap T_{G r, \overline{e_{34}}}$ is the projective space with coordinates $p_{13}, p_{14}, p_{23}, p_{24}$ and $C$ lies on the quadric surface $G r \cap P(C)$ given by $-p_{13} p_{24}+p_{14} p_{23}=0$. Identifying $G r \cap P(C)$ with $\mathbb{P}^{1} \times \mathbb{P}^{1}$ leads to $C \subset \mathbb{P}^{1} \times \mathbb{P}^{1}$ of bi-degree $\left(a_{1}, a_{2}\right)$ with $a_{1}+a_{2}=d$.

Consider the rational map $f: \mathbb{P}(V) \cdots \rightarrow \mathbb{P}^{1} \times \mathbb{P}^{1}$, given by $\left(t_{1}, t_{2}, t_{3}, t_{4}\right) \mapsto$ $\left(\left(t_{1}, t_{2}\right),\left(t_{3}, t_{4}\right)\right)$, which is defined outside the two lines $\bar{w}_{1}, \bar{w}_{2}$. The surface $S$ is the Zariski closure of $f^{-1}(C)$ and so the equation $F$ of $S$ is bi-homogeneous and coincides with the equation for $C \subset \mathbb{P}^{1} \times \mathbb{P}^{1}$. The other statements of the lemma are easily verified.

Observation 1.11 $\operatorname{dim} P(C)=3$ and $P(C)$ in a single tangent space of Gr. For a suitable basis of $V$ the projective subspace $P(C) \subset T_{G r}, \overline{e_{12}}$ is given by the equations $p_{34}=$ $0, p_{13}+p_{24}=0$ and $p_{12}, p_{13}, p_{14}, p_{23}$ are the homogeneous coordinates of $P(C)$. Further $G r \cap P(C)$ is the cone with equation $p_{13}^{2}+p_{14} p_{23}=0$ with vertex $\overline{e_{12}}$. Since $C$ lies on this cone we have a rational map $f: C \cdots \rightarrow E:=\left\{p_{13}^{2}+p_{14} p_{23}=0\right\}$. This map can be identified with the rational map $C \cdots \rightarrow \overline{e_{12}}$, given by $\bar{w} \mapsto \bar{w} \cap \overline{e_{12}}$. The rational map $f$ is 
a morphism if $\overline{e_{12}} \notin C$ or if $\overline{e_{12}} \in C$ and this is a regular point of $C$. Let $e$ be the degree of the morphism $f$.

In case $\overline{e_{12}} \notin C$, take two unramified points $e_{1}, e_{2} \in E$ and the plane through the corresponding two lines through $\overline{e_{12}}$. This plane meets $C$ in $2 e$ points. Hence $d=2 e$. In case $\overline{e_{12}} \in C$ and is not a singular point, the same reasoning yields $d-1=2 e$.

It seems difficult to investigate the possibilities for general $d$. The cases $d=3$ and $d=4$ will be presented later on.

\subsection{The vector bundle $B$ on $C^{\text {norm }}$}

Let again $d \geq 3$ denote the degree of the ruled surface $S$ and let $C \subset G r$ be the corresponding curve. Put

$$
B:=\left\{(p, v) \in C^{n o r m} \times V \mid p \mapsto \bar{w} \in C, w \wedge v=0\right\} \subset C^{\text {norm }} \times V .
$$

This is a (geometric) vector bundle of rank two on $C^{\text {norm }}$. We will identify $B$ with its sheaf of sections. We note that $\operatorname{Proj}(B)=\tilde{\tilde{S}}$. The line bundle $\Lambda^{2} B$ on $C^{\text {norm }}$ is the pullback of the restriction of $O_{\mathbb{P}\left(\Lambda^{2} V\right)}(-1)$ to $C$ and has therefore degree $-d$. The vector space $H^{0}\left(C^{\text {norm }}, B\right)=0$, otherwise all the lines of $C$ pass through one point and $S$ is a cone.

The vector bundle $B$ is an important tool in case $C^{\text {norm }}$ has genus 0 .

For the case $d=3$ it is easily seen that $C^{\text {norm }}$ has genus 0 . Let $t$ parametrize $C^{\text {norm }}$. One has $B \cong O_{C^{n o r m}}(-1) \oplus O_{C^{n o r m}}(-2)$ since $B$ has no holomorphic sections. In particular, $\tilde{\tilde{S}}$ is isomorphic to $\mathbb{P}^{2}$ with one point blown up (see [8], V, Example 2.11.5).

The sections of $B$ with a pole of order 1 at $t=\infty$ are $\mathbb{C} a$ and those with a pole of order $\leq 2$ at $t=\infty$ are $\mathbb{C} a+\mathbb{C} b$. By choosing a suitable basis of $V$ one can normalize to the following two cases:

$$
a(t)=(1, t, 0,0), b(t)=\left(0,0,1, t^{2}\right) \text { and } a(t)=(1, t, 0,0), b(t)=\left(0,1, t, t^{2}\right) .
$$

The surface $S$ consists of the lines connecting the pairs of points $\{a(t), b(t)\}$ for $t \in \mathbb{P}^{1}$. We find the equations $t_{1}^{2} t_{4}-t_{2}^{2} t_{3}=0$ and $t_{3}^{3}+t_{4}\left(t_{1} t_{4}-t_{2} t_{3}\right)=0$ and the classification of the ruled cubic surfaces over, say, $\mathbb{C}$.

In Sect. 2 we follow another method to obtain the classification of ruled cubic surfaces over any field and compare this with a method of Dolgachev.

Ford $=4$ and assuming that $C^{\text {norm }}$ has genus 0 , there are ( $B$ has no holomorphic sections) two possibilities for the vector bundle $B$, namely:

$$
\begin{aligned}
& B \cong O_{C^{n o r m}}(-1) \oplus O_{C^{n o r m}}(-3) \text { and } \underset{\tilde{S}}{\tilde{n}} \text { is the Hirzebruch surface } \Sigma_{2}, \\
& B \cong O_{C^{n o r m}}(-2) \oplus O_{C^{n o r m}}(-2) \text { and } \tilde{\tilde{S}} \text { is } \mathbb{P}^{1} \times \mathbb{P}^{1} .
\end{aligned}
$$

In the first case, overlooked by Cremona, there are meromorphic sections $a(t), b(t)$ with degrees 1 and 3 and the surface $S$ is the union of the lines connecting the pairs $\{a(t), b(t)\}$. In the second case, the meromorphic sections $a(t), b(t)$ both have degree 2 and the surface $S$ is determined in a similar way. As explained in the Introduction, this is Cremona's method to obtain ruled quartic surfaces. The method of Cayley can be interpreted as taking three sections of the vector bundle $B(d)$ for a certain values of $d \geq 1$.

Normalizing sections of $B$ with poles of order 1,2,3 at $t=\infty$, by a choice of the basis of $V$ and possibly changing $t$, we will arrive in Sect. 3.1 at a classification of the corresponding ruled quartic surfaces.

If the genus of $C^{\text {norm }}$ is 1 , the vector bundle $B$ is not helpful for the computation. However, $B$ and also $\tilde{\tilde{S}}=\operatorname{Proj}(B)$ will be identified. 
1.4 The possibilities for the singular locus

It is helpful for the classification of the ruled surfaces to consider $Q:=S \cap H$ with $H \subset \mathbb{P}(V)$ a general plane. By Bertini's theorem, $Q$ is an irreducible reduced curve of degree $d$. The morphism $C \rightarrow Q$, given by $\bar{w} \in C \mapsto \bar{w} \cap H \in Q$, is birational. Thus $C^{\text {norm }}$ is the normalization of $Q$. The singular locus of $S$ is written as a union of its irreducible components $C_{i}, i=1, \ldots, s$ of degree $d_{i}$ and generic multiplicity $m_{i} \geq 2$. The curve $Q$ meets every $C_{i}$ in $d_{i}$ points with multiplicity $m_{i}$. For every singular point $q \in Q$ one defines a number $\delta_{q}$ which is the sum of the integers $\frac{k(k-1)}{2}$ taken over the multiplicities $k$ of $q$ itself and of all the singular points that occur in the successive blow ups of $q$. The Plücker formula states that the genus of the normalization $C^{\text {norm }}$ of $Q$ is $\frac{(d-1)(d-2)}{2}-\sum \delta_{q}$.

For $d=3$, there is a single singular point $q$ and $\delta_{q}=1$ (and $q$ is a node or cusp). The singular locus is described by $s=1, d_{1}=1, m_{1}=2$.

For $d=4$, there are more possibilities. We adopt the terminology of the standard reference [1], p.62. The singularities of a simple plane curve (i.e., reduced, multiplicity $\leq 3$ and in the blow ups there are only singularities of multiplicity $\leq 3$ ) are classified by formal standard equations $F \in K[[x, y]]$. The condition that $Q$ is irreducible, has degree 4 and the genus of its normalization $C^{\text {norm }}$ is 0 or 1, leads to the list of possibilities (with their symbols or names):

$$
\begin{gathered}
\text { for } m=2: A_{2}: x^{2}-y^{2}, \delta=1 ; A_{3}: x^{2}-y^{3}, \delta=1 ; A_{4}: x^{2}-y^{4}, \delta=2 ; \\
\text { for } m=3: D_{4}: y\left(x^{2}-y^{2}\right), \delta=3 ; D_{5}: y\left(x^{2}-y^{3}\right), \delta=3 ; \quad E_{6}: x^{3}-y^{4}, \delta=3 \\
\quad \text { and the last case } E_{7}: x\left(x^{2}-y^{3}\right), \text { which is ruled out by } \delta=4 .
\end{gathered}
$$

The inequalities $\sum_{i=1}^{s} d_{i} \frac{m_{i}\left(m_{i}-1\right)}{2} \leq \sum \delta_{q} \leq \frac{(4-1)(4-2)}{2}$ lead to a list of possibilities for the singular locus, again with Cayley's convention that $d^{m}$ stands for an irreducible curve of degree $d$ and with multiplicity $m$ and 'int' meaning intersection lines: $1^{2} ; 1^{3} ; 2^{2} ; 3^{2} ; 1^{2}, 1^{2}$ int; $1^{2}, 1^{2} ; 1^{2}, 2^{2} ; 1^{2}, 1^{2}, 1^{2}$.

\subsection{The reciprocal of a ruled surface}

As before, $V$ is a vector space of dimension 4 over a field $K$. One identifies $\Lambda^{4} V$ with $K$. The non degenerate symmetric bilinear form on $\Lambda^{2} V$, given by $\left(w_{1}, w_{2}\right)=w_{1} \wedge w_{2} \in \Lambda^{4} V=K$, yields an isomorphism $f: \Lambda^{2} V \rightarrow \Lambda^{2} V^{*}=\left(\Lambda^{2} V\right)^{*}$ by $f\left(w_{1}\right)\left(w_{2}\right)=w_{1} \wedge w_{2} \in K$. This isomorphism maps decomposable vectors of $\Lambda^{2} V$ to decomposable vectors of $\Lambda^{2} V^{*}$.

Indeed, consider $f\left(v_{1} \wedge v_{2}\right)$. Let $v_{1}, v_{2}, v_{3}, v_{4}$ be a basis of $V$. The kernel of $f\left(v_{1} \wedge v_{2}\right)$ has basis $v_{1} \wedge v_{2}, v_{1} \wedge v_{3}, v_{1} \wedge v_{4}, v_{2} \wedge v_{3}, v_{2} \wedge v_{4}$. Let $\ell_{1}, \ell_{2}$ be a basis of $\left(V / K v_{1}+K v_{2}\right)^{*} \subset V^{*}$. Then $\ell_{1} \wedge \ell_{2}$ has the same basis vectors in the kernel. Hence $f\left(v_{1} \wedge v_{2}\right)$ is a multiple of $\ell_{1} \wedge \ell_{2}$.

Thus $f$ induces an isomorphism $\tilde{f}: \operatorname{Gr}(2, V) \rightarrow \operatorname{Gr}\left(2, V^{*}\right)$. Let $P \subset V$ be a 2-dimensional subspace. Then $\tilde{f}(P)$ is the 2-dimensional subspace $(V / P)^{*}$ of $V^{*}$. For 2-dimensional subspaces $P_{1}, P_{2} \subset V$ with $P_{1} \cap P_{2} \neq 0$ one has $\left(V / P_{1}\right)^{*} \cap\left(V / P_{2}\right)^{*} \neq 0$. This also follows from the formula $f\left(w_{1}\right) \wedge f\left(w_{2}\right)=w_{1} \wedge w_{2}$ for any $w_{1}, w_{2} \in \Lambda^{2} V$ (for a suitable identification of $\Lambda^{4} V^{*}$ with $K$ ).

Any 1-dimensional subspace $L \subset V$ determines the plane in $\operatorname{Gr}(2, V)$ consisting of all 2-dimensional $P \subset V, P \supset L$ (an $\omega$-plane in [7]). The image of this plane under $\tilde{f}$ is the plane in $\operatorname{Gr}\left(2, V^{*}\right)$ consisting of all 2-dimensional $Q \subset(V / L)^{*} \subset V^{*}$. Since the latter is a plane of the 'opposite type' (a $\rho$-plane in [7]), there is no isomorphism $V \rightarrow V^{*}$ inducing $\tilde{f}$. 
Let $e_{1}, \ldots, e_{4}$ denote a basis of $V$ and $e_{1}^{*}, \ldots, e_{4}^{*}$ the dual basis of $V^{*}$. Then $e_{i j}:=$ $e_{i} \wedge e_{j}, i<j$ is a basis of $\Lambda^{2} V$ and $e_{i j}^{*}=e_{i}^{*} \wedge e_{j}^{*}, i<j$ is a basis of $\Lambda^{2} V^{*}$. From the Plücker coordinates $\left\{p_{i j}\right\}$ of a line $\bar{w} \in G r(2, V)$ one easily finds the Plücker coordinates of $f(\bar{w}) \in \operatorname{Gr}\left(2, V^{*}\right)$ by the identities

$f\left(e_{12}\right)=e_{34}^{*} ; f\left(e_{13}\right)=-e_{24}^{*} ; f\left(e_{14}\right)=e_{23}^{*} ; f\left(e_{23}\right)=e_{14}^{*} ; f\left(e_{24}\right)=-e_{13}^{*} ; f\left(e_{34}\right)=e_{12}^{*}$.

Let an irreducible ruled surface $S \subset \mathbb{P}(V)$ (of degree $d$ ) be given by an irreducible curve $C \subset G r(2, V)$ of degree $d$. Consider a non singular point $s \in S$ lying on a single line $\ell$ of the surface. The tangent plane $T_{S, s}$ contains the line $\ell$. The same holds for the tangent planes $T_{S, s^{\prime}}$ for almost all points $s^{\prime} \in \ell$. Since $T_{S, s^{\prime}}$ cannot be all equal, the reciprocal (or dual) surface contains all planes $H \supset \ell$. It now follows that the reciprocal surface $\check{S} \subset \mathbb{P}\left(V^{*}\right)$ is ruled, the corresponding curve in $\operatorname{Gr}\left(2, V^{*}\right)$ is $f(C)$. It also has degree $d$ since the degree of the curve $f(C)$ is $d$.

Using Plücker coordinates one easily finds $\check{S}$. Another useful computation of the reciprocal surface is the following.

Consider $\tilde{S}=\{(\bar{w}, \bar{v}) \in C \times \mathbb{P}(V) \mid w \wedge v=0\} \rightarrow S \subset \mathbb{P}(V)$ and a non singular point $\bar{v}_{0} \in S$ and the non singular point $\bar{w}_{0} \in C$ with $w_{0} \wedge v_{0}=0$. (We note that the tangent plane of $\tilde{S}$ at the point $\left(\bar{w}_{0}, \bar{v}_{0}\right)$ is mapped isomorphically to the tangent plane of $S$ at the point $\bar{v}_{0}$. The first tangent plane is the product of the tangent line of $C$ at the point $\bar{w}_{0}$ and the line $\bar{w}_{0}$ ).

Let a (local) parametrization $t \mapsto w(t)$ for $C$ be given, such that $w_{0}=w\left(t_{0}\right)$. Choose a decomposition $w(t)=a(t) \wedge b(t)$, locally at $t_{0}$. Then $v_{0}=s_{0} a\left(t_{0}\right)+\left(1-s_{0}\right) b\left(t_{0}\right)$ and $S$ has the local parametrization $(t, s) \mapsto s a(t)+(1-s) b(t)$. The linearization of this, i.e.,

$$
v_{0}+\left(s-s_{0}\right)\left(a\left(t_{0}\right)-b\left(t_{0}\right)\right)+\left(t-t_{0}\right)\left(s_{0} a^{\prime}\left(t_{0}\right)+\left(1-s_{0}\right) b^{\prime}\left(t_{0}\right)\right),
$$

is a parametrization of the tangent plane $T_{S, \bar{v}_{0}}$. This corresponds with the 3-dimensional subspace of $V$ with basis

$$
v_{0}, v_{0}+a\left(t_{0}\right)-b\left(t_{0}\right), v_{0}+s_{0} a^{\prime}\left(t_{0}\right)+\left(1-s_{0}\right) b^{\prime}\left(t_{0}\right) .
$$

The exterior product $a\left(t_{0}\right) \wedge b\left(t_{0}\right) \wedge\left(s_{0} a^{\prime}\left(t_{0}\right)+\left(1-s_{0}\right) b^{\prime}\left(t_{0}\right)\right)$ of these vectors is an element of $\Lambda^{3} V=V^{*}$. This defines a point in $\mathbb{P}\left(V^{*}\right)$ corresponding to the tangent plane $T_{S, \bar{v}_{0}}$. The reciprocal surface $\breve{S}$ consists of all these points. In varying $s_{0}$ one finds a line on $\breve{S}$, through the points $a\left(t_{0}\right) \wedge b\left(t_{0}\right) \wedge a^{\prime}\left(t_{0}\right)$ and $a\left(t_{0}\right) \wedge b\left(t_{0}\right) \wedge b^{\prime}\left(t_{0}\right)$. By varying $t_{0}$ one obtains $\check{S}$.

For the two cases of ruled quartic surfaces $S$ with genus 1 , it is easily seen that $\breve{S} \cong S$. For the ruled quartic surfaces cases of genus 0 there are explicit global expressions $w(t)=$ $a(t) \wedge b(t)$ (with $t \in \mathbb{P}^{1}$ ) and the above can be used for the computation of $\check{S}$.

\section{Ruled surfaces of degree 3}

Here we give the classification over an arbitrary field $K$. The singular locus of $S$ is a line, $C^{\text {norm }}$ has genus 0 and $\operatorname{dim} P(C)=3$. This implies that $C=C^{\text {norm }}$ is the twisted cubic curve in $P(C)$.

In the first case $P(C)$ lies in two tangent spaces at the points $\bar{w}_{1}, \bar{w}_{2} \in G r$. From Lemma 1.10 we conclude that $S$ is given by a bi-homogeneous equation $F$ in the pairs of variables $t_{1}, t_{2}$ and $t_{3}, t_{4}$ of bi-degree $(2,1)$, corresponding to a morphism $f: \bar{w}_{1} \rightarrow \bar{w}_{2}$ of degree 2. The line $\bar{w}_{1}$ is non singular and a 'directrix'. The line $\bar{w}_{2}$ is the singular locus. Further $\tilde{C} \backslash C=\left\{\bar{w}_{1}, \bar{w}_{2}\right\}$. 
If the field $K$ has characteristic $\neq 2$, then $C, P(C), \bar{w}_{1}, \bar{w}_{2}$ are all defined over $K$ and can be put in a standard form. The morphism $f$ is defined over $K$. The ramification points of $f$ are either both in $K$ or belong to a quadratic extension of $K$ and are conjugated. Explicit expressions for $f$ can be chosen to be $\left(t_{1}, t_{2}, 0,0\right) \mapsto\left(0,0, t_{2}^{2},-t_{1}^{2}\right)$ and $\left(t_{1}, t_{2}, 0,0\right) \mapsto$ $\left(0,0, a t_{1}^{2}+t_{2}^{2},-t_{1} t_{2}\right)$ with $a \in K^{*}$, not a square.

If the field $K$ has characteristic 2 , then $f$ is either separable and has one point of ramification (and has standard form $\left.\left(t_{1}, t_{2}, 0,0\right) \mapsto\left(0,0, t_{2}^{2}+t_{1} t_{2}, t_{1}^{2}\right)\right)$, or $f$ is inseparable (and has standard form $\left.\left(t_{1}, t_{2}, 0,0\right) \mapsto\left(0,0, t_{2}^{2}, t_{1}^{2}\right)\right)$.

In the second case $P(C)$ lies in only one tangent space, namely at the point $\bar{w}_{0}$ which is the singular line of $S$. Then $C$ lies on the quadratic cone in $P(C)$ and $\bar{w}_{0} \in C$. In this case $\tilde{C}=C$. Now $C$ and $S$ can be put into a standard form. We arrive at the following result.

Proposition 2.1 The standard equations for ruled cubic surfaces $S / K$, which are not cones, are the following

(1) $t_{3} t_{1}^{2}+t_{4} t_{2}^{2}=0$. If char $K=2$, then there are no twist. For char $K \neq 2$ the twists are $t_{3}\left(t_{1} t_{2}\right)+t_{4}\left(a t_{1}^{2}+t_{2}^{2}\right)=0$ with $a \in K^{*}$ not a square.

(2) $t_{3} t_{1} t_{2}+t_{4} t_{1}^{2}+t_{2}^{3}=0$ (there are no twists).

(3) $t_{3} t_{1}^{2}+t_{4}\left(t_{2}^{2}+t_{1} t_{2}\right)=0$ if char $K=2$ (there are no twists).

The curves $C$ for (1) and (2) are in parameter form

$$
\begin{aligned}
& p_{12}=0, p_{13}=-t^{2}, p_{14}=1, p_{23}=-t^{3}, p_{24}=t, p_{34}=0 \text { and } \\
& p_{12}=0, p_{13}=t^{3}, p_{14}=t^{2}, p_{23}=-t^{2}, p_{24}=-t, p_{34}=-1 .
\end{aligned}
$$

The above equations for $S$ are derived in an elegant way by I. Dolgachev [6], using only the information that the singular locus of $S$ is a line with multiplicity 2.

For $K=\mathbb{R}$, there are three types of cubic ruled surfaces (omitting cones). The plaster models VII, nr. 20-23 and the string models XVIII, nr. 1-4, in part I of the Schilling catalogue, represent equations (1) and (2).

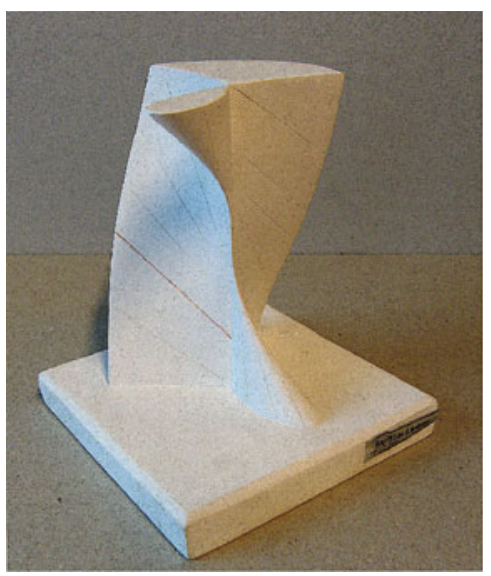

Series VII 23

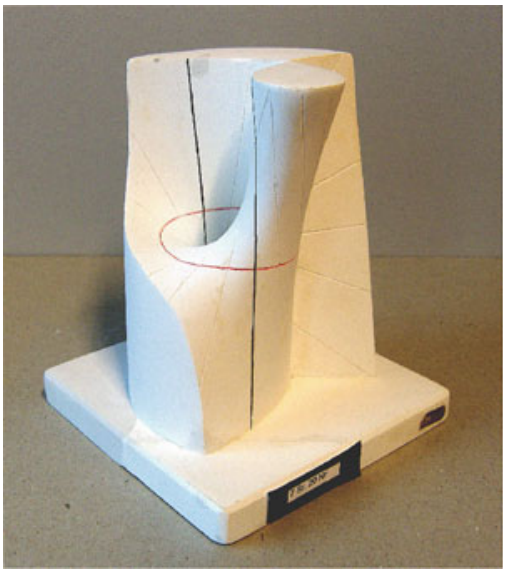

Series VII 20

\section{Ruled surfaces of degree 4}

The base field $K$ is supposed to be algebraically closed. The only role that the characteristic of $K$ plays is in the classification of the morphisms $\mathbb{P}^{1} \rightarrow \mathbb{P}^{1}$ of degree 2 and 3 . For convenience 
we suppose that $K$ has characteristic 0 or $>3$. We need both the vector bundle $B$ and the possibilities for the singular locus in order to find all cases and to verify the computations by comparison.

\subsection{Classification of $S$, using the vector bundle $B$}

\subsection{1 $C^{\text {norm }}$ of genus 0 and $B$ of type $-1,-3$}

Choose a parameter $t$ for $C^{\text {norm }} \cong \mathbb{P}^{1}$ and let $p$ be the pole of $t$. Then $H^{0}\left(C^{\text {norm }}, B([p])\right)$ has basis $a$ and $H^{0}\left(C^{n o r m}, B(3 \cdot[p])\right)$ has basis $a, t a, t^{2} a, b$. Now $a, b \in K[t] \otimes_{K} V$ have degrees 1 and 3 . We note that $b$ is not unique and can be replaced by $\mu b+\lambda_{0} a+\lambda_{1} t a+\lambda_{2} t^{2} a$ with $\mu \neq 0$.

We start with some observations. First we recall that $S$ consists of the lines through the pairs of points $\bar{a}(t), \bar{b}(t) \in \mathbb{P}(V)$ with $t \in \mathbb{P}^{1}$. The (unique) morphism $\bar{a}: C^{\text {norm }} \rightarrow \mathbb{P}(V)$ is not constant since $S$ is not a cone. Let $W \subset V$ be the 2-dimensional subspace defined by $a \in K[t] \otimes W$. Let $b^{\prime}$ be the image of $b$ in $K[t] \otimes(V / W)$. The points of $\mathbb{P}(V / W)$ can be identified with the planes in $\mathbb{P}(V)$ containing the line $\mathbb{P}(W)$. The (unique) morphism $\overline{b^{\prime}}: C^{\text {norm }} \rightarrow \mathbb{P}(V / W)$ is clearly not constant and its degree can be 1,2 or 3 .

Now we want to derive 'normal forms' for $a$ and $b$. This means that we make choices for $b, t$ and a basis $e_{1}, e_{2}, e_{3}, e_{4}$ of $V$ such that the formulas for $a$ and $b$ (as 4-tuples) are as simple as possible. This is done case by case. For the first three cases the details are presented and for the later cases we restrict to writing down a 'normal form'.

The Plücker coordinates of the line through explicit points $a(t), b(t)$ are easily computed and this yields $C \subset G r$ in parametrized form. From this one deduces $\operatorname{dim} P(C)$, possible singularities of $C$ and the relation of $C$ w.r.t. the tangent spaces of $G r$. The reciprocal surface (needed for the comparison with Cremona's list) is computed by the method of $\S 1.5$, again using $a(t), b(t)$. For some cases (especially when the singular locus is $3^{2}$ ), the equation of the surface $S$ (done by a MAPLE computation) is rather long and uninteresting. We avoid this and compute the singular locus by other means.

Suppose that the degree of $\overline{b^{\prime}}$ is 3 . The possibilities for the ramification indices of $\overline{b^{\prime}}$ are: (a) 3, 3, (b) 3,2, 2 or (c) 2, 2, 2, 2. A choice of $t$, parametrizing $C^{\text {norm }}$, and a choice of the basis $\bar{e}_{3}, \bar{e}_{4}$ of $V / W$ brings $b^{\prime}$ into a standard form

$$
\left(1, t^{3}\right),\left(1, t^{2}(t+1)\right) \text {, or }\left(t-\mu,(2 \mu-1) t^{3}+(2-3 \mu) t^{2}\right) \text { with } \mu \neq 0,1,1 / 2 \text {. }
$$

In the last case the 4 ramification points are $0,1, \infty, \frac{\mu}{2 \mu-1}$.

Preimages $e_{3}, e_{4}$ of $\bar{e}_{3}, \bar{e}_{4}$ and a basis $e_{1}, e_{2}$ of $W$ can be chosen such that $a=(1, t, 0,0)$, $b=\left(b_{1}, b_{2}, b_{3}, b_{4}\right) ;\left(b_{3}, b_{4}\right)$ as above and $\max \left(\operatorname{deg} b_{1}, \operatorname{deg} b_{2}\right) \leq 2$. Then $b-b_{1} \cdot a$ produces a new $b=\left(0, b_{2}, b_{3}, b_{4}\right)$. There are two cases:

Number 1 (a,b,c) $\quad b_{2}=0$. One finds: $P(C)=T_{G r, \overline{e_{12}}} \cap T_{G r, \overline{e_{34}}} ; \overline{e_{12}}, \overline{e_{34}} \notin C ; C$ is non singular; equation $t_{1}^{3} b_{3}\left(\frac{t_{2}}{t_{1}}\right) t_{4}-t_{1}^{3} b_{4}\left(\frac{t_{2}}{t_{1}}\right) t_{3}=0$; the singular locus of $S$ is the line $\overline{e_{34}}$ with multiplicity 3 . Then $1^{3}$ and Cremona 9 .

Number 2 (a,b,c) $\quad b_{2} \neq 0$. One finds: $C$ non singular, $P(C)=T_{G r, \overline{e_{12}}}$ and $\overline{e_{12}} \notin C$. A direct computation of the equation seems difficult. The points of the line through $a(t)$ and $b(t)$ can be written as $\left(1, t+\lambda \frac{b_{2}}{b_{3}}(t), \lambda, \lambda \frac{b_{4}}{b_{3}}(t)\right)$. The intersection of two lines on $S$ is given by the equality

$$
\left(1, t+\lambda \frac{b_{2}}{b_{3}}(t), \lambda, \lambda \frac{b_{4}}{b_{3}}(t)\right)=\left(1, s+\lambda \frac{b_{2}}{b_{3}}(s), \lambda, \lambda \frac{b_{4}}{b_{3}}(s)\right) \text {, with } s \neq t .
$$


This leads to the result that the 'double curve' and thus also the singular locus is a twisted cubic curve. Then $3^{2}$ and Cremona 7 .

For the next cases we start with a basis $e_{1}, e_{2}, e_{3}, e_{4}$ of $V$, such that $e_{1}, e_{2}$ is a basis of $W$. Thus $a=\left(a_{1}, a_{2}, 0,0\right)$ and $b=\left(b_{1}, b_{2}, b_{3}, b_{4}\right)$. We modify this basis and $t$ in order to obtain a 'normal form'.

Number 3 (a,b) Suppose that $\max \left(\operatorname{deg} b_{3}, \operatorname{deg} b_{4}\right)=2$ and g.c.d. $\left(b_{3}, b_{4}\right)=1$. Since $\overline{b^{\prime}}$ has degree 2 , one can normalize $\left(b_{3}, b_{4}\right)=\left(t^{2}, 1\right)$. Further $a$ is normalized to $\left(a_{1}, 1,0,0\right)$ with $\operatorname{deg} a_{1}=1$. After subtracting from $b$ a suitable $\left(*+* t+* t^{2}\right) a$ one obtains that $b_{1}$ is a constant. Base changes $e_{3} \mapsto e_{3}+* e_{1}+* e_{2} ; e_{4} \mapsto e_{4}+* e_{1}+* e_{2}$ eliminate the terms $* t^{2}$ and $*$ from $b_{1}$ and $b_{2}$. Finally, after multiplying $e_{1}, \cdots, e_{4}$ and $t$ by constants one obtains a normalization $a=(t+\beta, 1,0,0)$ and $b=\left(0, t^{3}+\alpha t, t^{2}, 1\right)$. Equation

$$
t_{3} t_{4}\left(t_{2}-\beta\left(t_{3}+\alpha t_{4}\right)\right)^{2}-\left(t_{3}\left(t_{3}+\alpha t_{4}\right)-\beta t_{2} t_{4}+t_{1} t_{4}\right)^{2}=0 .
$$

One has $P(C)=T_{G r, \overline{e_{12}}}$ and $\overline{e_{12}} \in C$. The singular locus is the union of the line $\overline{e_{12}}$ and the conic $t_{2}-\beta\left(t_{3}+\alpha t_{4}\right)=0, t_{3}\left(t_{3}+\alpha t_{4}\right)-\beta t_{2} t_{4}+t_{1} t_{4}=0$. Then $1^{2}, 2^{2}$ and Cremona 4. The degree morphism $C \rightarrow D$, where $D$ denotes the conic, has two points of ramification. The point $L \cap D$ is a ramification point on $D$ if and only if $\beta=0$. We consider this as two distinct cases.

Number 4 Suppose $\max \left(\operatorname{deg} b_{3}, \operatorname{deg} b_{4}\right)=2$ and g.c.d. $\left(b_{3}, b_{4}\right)$ has degree 1 .

A normalization is $a=(t, 1,0,0), b=\left(0, t^{3}+\alpha, t(t+\beta), t+\beta\right)$ with g.c.d. $\left(t^{3}+\right.$ $\alpha, t+\beta)=1$. The equation is

$$
t_{1} t_{4}^{2}\left(t_{3}+\beta t_{4}\right)-t_{2} t_{3} t_{4}\left(t_{3}+\beta t_{4}\right)+\alpha t_{3} t_{4}^{3}+t_{3}^{4}=0 .
$$

Further $\overline{e_{12}} \in C$ is a node (for $t=\infty, t=-\beta$ ), $\operatorname{dim} P(C)=3$ and $P(C)$ lies in only one tangent space $T_{G r, \overline{e_{12}}}$. Then $1^{3}$ and Cremona 10 .

Number 5 Suppose that $\max \left(\operatorname{deg} b_{3}, \operatorname{deg} b_{4}\right)=1$.

A normalization is $a=(t, 1,0,0), b=\left(0, t^{3}+\alpha t^{2}, t, 1\right)$. The equation is

$$
t_{1} t_{4}^{3}-t_{2} t_{3} t_{4}^{2}+\alpha t_{3}^{3} t_{4}+t_{3}^{4}=0 .
$$

Further $\overline{e_{12}} \in C$ is a cusp (for $t=\infty$ ), $\operatorname{dim} P(C)=3$ and $P(C)$ lies in only one tangent space, namely $T_{G r, \overline{e_{12}}}$. Then $1^{3}$ and Cremona 10 .

Finally, the omitted cases for $\left(b_{3}, b_{4}\right)$ can be reduced to the above. Indeed:

For $\max \left(\operatorname{deg} b_{3}, \operatorname{deg} b_{4}\right)=3$ and g.c.d. $\left(b_{3}, b_{4}\right)$ of degree 1 , a normalization is $a=(1, t, 0,0), b=\left(b_{1}, b_{2}, t, t(t+\mu)^{2}\right)$. Replacing $t$ by $s^{-1}$ and multiplying by $s^{3}$ yields $a=(s, 1,0,0), \quad b=\left(s^{3} b_{1}\left(s^{-1}\right), s^{3} b_{2}\left(s^{-1}\right), s^{2},(1+\mu s)^{2}\right)$. Thus reduction to $\max \left(\operatorname{deg} b_{3}, \operatorname{deg} b_{4}\right)=2$.

For $\max \left(\operatorname{deg} b_{3}, \operatorname{deg} b_{4}\right)=3$ and g.c.d. $\left(b_{3}, b_{4}\right)$ of degree 2 , a normalization is $a=$ $(1, t, 0,0), b=\left(b_{1}, b_{2}, t(t+\mu), t(t+\mu)(t+\lambda)\right)$. Replacing $t$ by $s^{-1}$ and multiplying by $s^{3}$ gives a reduction to $\max \left(\operatorname{deg} b_{3}, \operatorname{deg} b_{4}\right)=2$.

\subsection{2 $C^{\text {norm }}$ of genus 0 and $B$ of type $-2,-2$}

$V, t, p$ have the meaning of $\S 3.1 .1$. The vector space $H^{0}\left(C^{n o r m}, B(2[p])\right)$ has dimension 2 and consists of elements in $K[t] \otimes V$ of degree $\leq 2$ and the only element of degree $\leq 1$ is 0 . We are interested in lines $K a \subset H^{0}\left(C^{n o r m}, B(2[p])\right)$ such that $a \in K[t] \otimes W$ with $\operatorname{dim} W=2$. We note that for such $K a$, the image of the corresponding morphism $\bar{a}: C^{\text {norm }} \rightarrow \mathbb{P}(V)$ is a line. For the other lines $K a$, the image of $\bar{a}$ is a conic. 
Suppose that there are two such lines $K a$ and $K b$ in $H^{0}\left(C^{n o r m}, B(2[p])\right)$.

One can normalize to $a=\left(a_{1}, a_{2}, 0,0\right), b=\left(0,0, b_{3}, b_{4}\right)$. The two morphisms $\bar{a}, \bar{b}$ : $C^{\text {norm }} \rightarrow \mathbb{P}^{1}, t \mapsto\left(a_{1}, a_{2}\right)$ and $t \mapsto\left(b_{3}, b_{4}\right)$ have degree 2 and are distinct and their sets of ramification points can be either disjoint or have one point of intersection. Choosing $t$ leads to the following normalizations.

Number $6 a=\left(1, t^{2}, 0,0\right), b=\left(0,0,(t-1)^{2},\left(t-\lambda^{2}\right)^{2}\right)$. The singular locus consists of the lines $\overline{e_{12}}, \overline{e_{34}}$ and a third line corresponding to $t= \pm \lambda$. The morphism $C^{\text {norm }} \stackrel{(\bar{a}, \bar{b})}{\rightarrow} \mathbb{P}^{1} \times \mathbb{P}^{1}$ maps $t= \pm \lambda$ to the same point of $C$. Thus $C$ has a node, $P(C)=T_{G r, \overline{e_{12}}} \cap T_{G r, \overline{e_{34}}}$, singular locus $1^{2}, 1^{2}, 1^{2}$ and Cremona 5 .

Number $7 a=\left(1, t^{2}, 0,0\right), b=\left(0,0,1,(t-1)^{2}\right)$. The image $C$ of $C^{\text {norm }} \stackrel{(\bar{a}, \bar{b})}{\rightarrow} \mathbb{P}^{1} \times \mathbb{P}^{1}$ has a cusp corresponding to $t=\infty$. The singular locus consists of the lines $\overline{e_{12}}, \overline{e_{34}}$ and the line corresponding to $t=\infty$. Thus $P(C)=T_{G r, \overline{e_{12}}} \cap T_{G r, \overline{e_{34}}}$, singular locus $1^{2}, 1^{2}, 1^{2}$ and Cremona 5.

Suppose that there exists only one such line $\mathrm{Ka}$ in $H^{0}\left(C^{\text {norm }}, B(2[p])\right)$.

Normalize by $a=\left(1, t^{2}, 0,0\right), b=\left(b_{1}, b_{2}, b_{3}, b_{4}\right)$ with $\operatorname{deg} b_{2}<2$. The pair $\left(b_{3}, b_{4}\right)$ is, up to taking linear combinations, uniquely determined by the surface. The morphism $m: C^{\text {norm }} \rightarrow \mathbb{P}^{1}, t \mapsto\left(b_{3}: b_{4}\right)$ cannot be constant and has degree 1 or 2 . There are the following cases.

Number $\mathbf{8} \operatorname{deg} m=1,\left(b_{3}, b_{4}\right)=(1, t)$. Then $P(C)=T_{G r, \overline{e_{12}}}$ and $\overline{e_{12}} \notin C$. The equation is

$$
t_{1} t_{3} t_{4}^{2}-t_{2} t_{3}^{3}-t_{3}^{2} t_{4}^{2} b_{1}\left(\frac{t_{4}}{t_{3}}\right)+t_{3}^{4} b_{2}\left(\frac{t_{4}}{t_{3}}\right)=0 .
$$

Singular locus $1^{3}$ and Cremona 3 .

Number $9 \operatorname{deg} m=1,\left(b_{3}, b_{4}\right)=(t-\alpha, t(t-\alpha))$. Then $P(C)=T_{G r, \overline{e_{12}}}$ and $\overline{e_{12}} \in C$. The equation is

$$
t_{1}\left(t_{4}-\alpha t_{3}\right) t_{4}^{2}-t_{2} t_{3}^{2}\left(t_{4}-\alpha t_{3}\right)-t_{3}^{2} t_{4}^{2} b_{1}\left(\frac{t_{4}}{t_{3}}\right)+t_{3}^{4} b_{2}\left(\frac{t_{4}}{t_{3}}\right)=0 .
$$

Singular locus $1^{3}$ and Cremona 3 .

Number $10(\mathbf{a}, \mathbf{b}) \operatorname{deg} m=2$. If $t=0, \infty$ are the ramification points of $m$, then one normalizes to $\left(b_{3}, b_{4}\right)=\left(1, t^{2}\right)$. Then $\operatorname{dim} P(C)=3$ and $P(C)$ lies in only one tangent space, namely $T_{G r, \overline{e_{12}}}$, and $\overline{e_{12}} \notin C$. Write $b_{1}=b_{12} t^{2}+b_{11} t+b_{10}$ and $b_{2}=b_{21} t+b_{20}$. After a base change of the form $e_{3} \mapsto e_{3}+* e_{1}+* e_{2}, e_{4} \mapsto e_{4}+* e_{1}+* e_{2}$, one obtains $b_{1}=b_{11} t, b_{2}=b_{21} t$. Then $C$ has a node (case (a)) if $b_{21} b_{11} \neq 0$ and has a cusp otherwise (case (b)). Singular locus $1^{2}, 1^{2}$, int (i.e., intersecting lines) and Cremona 6.

Number $11 \operatorname{deg} m=2$. If $m: C^{\text {norm }} \rightarrow \mathbb{P}^{1}$ is ramified for, say, $t=1, \infty$, then one can normalize $\left.a=\left(1, t^{2}, 0,0\right), b=\left(b_{1}(t-1), b_{2}(t-1), 1,(t-1)^{2}\right)\right)$ with $b_{1}, b_{2} \in K$. The equation is

$$
t_{3} t_{4}\left(2 t_{1}+\left(b_{2}-b_{1}\right) t_{3}-b_{1} t_{4}\right)^{2}-\left(t_{2} t_{3}-t_{1} t_{3}-t_{1} t_{4}+2 b_{1} t_{3} t_{4}\right)^{2}=0 .
$$

The singular locus is the union of the line $L=\overline{e_{12}}$ and the conic $D$ given by $2 t_{1}+\left(b_{2}-\right.$ $\left.b_{1}\right) t_{3}-b_{1} t_{4}=0, t_{2} t_{3}-t_{1} t_{3}-t_{1} t_{4}+2 b_{1} t_{3} t_{4}=0 . P(C)=T_{G r, \overline{e_{12}}}, \overline{e_{12}} \notin C$, the image of $C \rightarrow L \times D, \bar{w} \mapsto(\bar{w} \cap L, \bar{w} \cap D)$ is a rational curve having a cusp. Singular locus $1^{2}, 2^{2}$ and Cremona 2. 
Number $12 \operatorname{deg} m=2$. If $m$ is ramified for, say, $t=1, \mu$ with $\mu \neq 0,1, \infty$, then one can normalize to $a=\left(1, t^{2}, 0,0\right) b=\left(b_{1}, b_{2},(t-1)^{2},(t-\mu)^{2}\right)$ with $b_{1}, b_{2} \in K$. One can replace $b$ by $b-b_{1} \cdot a$ and normalize further to $a=\left(1, t^{2}, 0,0\right), b=\left(0,1,(t-1)^{2},(t-\mu)^{2}\right)$. A somewhat long computation yields the equation

$$
\begin{aligned}
& 4 t_{3} t_{4}\left((\mu-1)^{2}\left(t_{2}-\mu t_{1}\right)-2 t_{3}-2 t_{4}\right)^{2}-\left((\mu-1)^{2}\left(-\mu^{2} t_{1} t_{3}-t_{1} t_{4}+t_{2} t_{3}+t_{2} t_{4}\right)\right. \\
& \left.-t_{3}^{2}-6 t_{3} t_{4}-t_{4}^{2}\right)^{2}=0 .
\end{aligned}
$$

The singular locus is the union of the line $L=\overline{e_{12}}$ and the conic $D$ given by the equations

$$
(\mu-1)^{2}\left(t_{2}-\mu t_{1}\right)-2\left(t_{3}+t_{4}\right)=0,(\mu-1)^{3} t_{1}\left(t_{3}-\mu t_{4}\right)-\left(t_{3}-t_{4}\right)^{2}=0 .
$$

Further $P(C)=T_{G r, \overline{e_{12}}}, \overline{e_{12}} \notin C$, the image of the morphism $C \rightarrow L \times D$ is a rational curve having a node. Singular locus $1^{2}, 2^{2}$ and Cremona 2.

Suppose that there is no such line $K a$ and that $P(C)$ lies in a tangent space.

The inclusion $P(C) \subset T_{G r, \overline{e_{12}}}$ yields a morphism $f: C^{\text {norm }} \rightarrow \overline{e_{12}}$ induced by $\overline{e_{12}} \neq \bar{w} \in C \mapsto \bar{w} \cap \overline{e_{12}}$. The degree of $f$ cannot be 1 . Indeed, if the degree of $f$ is 1 , then we may assume that $(1, t, 0,0)$ lies on $S$. Combining this with a non zero element $a \in H^{0}\left(C^{\text {norm }}, B(2[p])\right)$, one finds a surface of degree 3 instead of 4 .

The possibility that the degree of $f$ is 2 is excluded by the following reasoning. Let $t$ be a parameter for $C^{\text {norm }}$ and write $f=\left((\alpha t+\beta)^{2},(\gamma t+\delta)^{2}, 0,0\right)$. Let $a, b$ be a basis of $H^{0}\left(C^{\text {norm }}, B(2[p])\right)$. Then $\lambda_{0}(t) f=\lambda_{1}(t) a+\lambda_{2}(t) b$ holds for some $\lambda_{0}(t), \lambda_{1}(t), \lambda_{2}(t) \in$ $K[t]$ with $g . c . d .\left(\lambda_{1}(t), \lambda_{2}(t)\right)=1$.

The Plücker coordinates of $a \wedge b$ are polynomials in $t$ with g.c.d. 1 and maximal degree 4, since these parametrize $C$. The same holds for the Plücker coordinates of $f \wedge a$ and $f \wedge b$. The equality $\lambda_{0}(t) f \wedge a=-\lambda_{2}(t) \cdot a \wedge b$ implies that $\lambda_{0}(t)$ is a constant multiple of $\lambda_{2}(t)$. Similarly, $\lambda_{0}(t)$ is a constant multiple of $\lambda_{1}(t)$. We conclude that the $\lambda_{i}(t)$ are constant. Then $f \in H^{0}\left(C^{\text {norm }}, B(2[p])\right)$ and this contradicts the assumption.

Number $\mathbf{1 3}$ (a,b,c) If the degree of $f$ is 3 , then $\overline{e_{12}}$ has multiplicity 3 and the singular locus is $1^{3}$. As in the case of Number 2, there are three different possibilities for the ramification of $f$. One writes $f(t)=\left(c_{1}, c_{2}, 0,0\right)$ where $c_{1}, c_{2}$ are relatively prime polynomials in $t$ and, say, $\operatorname{deg} c_{1}<\operatorname{deg} c_{2}=3$. Let $a(t)=\left(a_{1}, a_{2}, a_{3}, a_{4}\right)$ be a non zero section of $B(2[p])$. An inspection of the Plücker coordinates of $f \wedge a$ implies that $\max \left(\operatorname{deg} a_{3}, \operatorname{deg} a_{4}\right) \leq 1$. Moreover $a_{3}, a_{4}$ are linearly independent. Thus we may normalize to $\left(a_{3}, a_{4}\right)=(1, t)$. Because $\overline{e_{12}}$ has multiplicity 3 , the equation for $S$ has the form $t_{1} A_{1}+t_{2} A_{2}+A_{3}=0$, where $A_{1}, A_{2}, A_{3}$ are homogeneous polynomials in $t_{3}$, $t_{4}$ of degrees 3,3 , 4. Substitution of $\left(\lambda c_{1}+a_{1}, \lambda c_{2}+a_{2}, 1, t\right)$ in this equation yields $c_{1}(t) A_{1}(1, t)+c_{2}(t) A_{2}(1, t)=0$ and we can normalize to $A_{1}(1, t)=c_{2}(t), A_{2}(1, t)=-c_{1}(t)$. In particular, g.c.d. $\left(A_{1}, A_{2}\right)=1$. Further $A_{3}(1, t)=-a_{1}(t) c_{2}(t)+a_{2}(t) c_{1}(t)$. The term $A_{3}$ cannot be made 0 by a transformation of the form $t_{1} \mapsto t_{1}+* t_{3}+* t_{4}, t_{2} \mapsto t_{2}+* t_{3}+* t_{4}$, since $P(C)$ does not lie in another tangent space. Therefore, $\max \left(\operatorname{deg} a_{1}, \operatorname{deg} a_{2}\right)=2$. Further $\operatorname{dim} P(C)=4$ and $\overline{e_{12}} \notin C$. One verifies that the equations belong to the case that $B$ has type $-2,-2$ by comparing with the cases $1^{3}$ where $B$ has type $-1,-3$. Further Cremona 8 .

Suppose that there is no such line and $P(C)$ does not lie in a tangent space. 
Number 14 Surface of species Cremona 1 and singular locus $3^{2} \cdot \operatorname{dim} P(C)=4$ and $C$ is non singular. According to Proposition 1.8, (3), $S$ does not contain a line. Then, because of Lemma 3.1 and $\S 1.4$, the singular locus of $S$ is the twisted cubic curve. In $\S 3.2 .4$ it is shown that this Number 14 consists of six distinct subclasses.

\subsubsection{The vector bundle B for a genus 1 curve $C^{\text {norm }}$}

The vector bundle $B$ does not provide information that leads to the possibilities for $S$. Instead, we use the information 3.2.5 and 3.2.7 of $\S 3.2$ and deduce the structure of the vector bundle $B$ on the genus 1 curve $C=C^{\text {norm }}$.

Number 15 Case $1^{2}$. The equation is $\left(t_{1} t_{4}-t_{2} t_{3}\right)^{2}+H\left(t_{3}, t_{4}\right)$, where $H$ is homogeneous of degree 4 and defines 4 distinct points on $\mathbb{P}\left(K e_{3}+K e_{4}\right)=\mathbb{P}^{1}$. We may suppose that these points are $0,1, \lambda, \infty$. The lines $\bar{w}(t) \in C$ on $S$ are computed to be the lines passing through the points $(1, t, 0,0)$ and $(0, y, 1, t)$, with $y^{2}=H(1, t)$. The genus one curve $C$ is made into an elliptic curve by the choice of the neutral element $e$ to correspond to $t=y=\infty$. We note that $\overline{e_{12}} \notin C ;(1, t, 0,0)$ is a section of $B(2[e])$ and $(0, y, 1, t)$ is a section of $B(3[e])$. Further $w(t)=(1, t, 0,0) \wedge(0, y, t, 1)=y e_{12}+e_{13}+t e_{14}+t e_{23}+t^{2} e_{24}+0 e_{34}$ is a section of $\Lambda^{2} B([4] e)$. Consider the exact sequence

$$
0 \rightarrow O_{C}(1, t, 0,0) \rightarrow B(2[e]) \rightarrow O_{C}(0,0,1, t) \rightarrow 0 .
$$

From $O_{C}(1, t, 0,0) \cong O_{C}(0,0,1, t) \cong O_{C}$ and $H^{0}(C, B(2[e]))=K(1, t, 0,0)$ one concludes that the sequence does not split. Therefore the ruled surface (in the modern sense) $\tilde{S} \rightarrow C$ corresponds to the unique indecomposable vector bundle on $C$ which is an extension of $O_{C}$ by $O_{C}$. (see [8]).

Number 16 Case $1^{2}, 1^{2}$. The equation $F$ for $S$ is bi-homogeneous of degree $(2,2)$ in the pairs of variables $t_{1}, t_{2}$ and $t_{3}, t_{4}$. The equation $F$ also defines a genus one curve $E \subset$ $\mathbb{P}\left(K e_{1}+K e_{2}\right) \times \mathbb{P}\left(K e_{3}+K e_{4}\right)$. Further $E \rightarrow C \subset G r$ is the isomorphism which sends $p \in E$ to the line through the points $\left(p r_{1}(p), 0,0\right)$ and $\left(0,0, p r_{2}(p)\right)$. The vector bundle $B$ is the direct sum of the line bundles $\mathcal{L}_{1}:=\left\{(\bar{w}, v) \mid \bar{w} \in C, v \in K e_{1}+K e_{2}, w \wedge v=0\right\}$ and $\mathcal{L}_{2}:=\left\{(\bar{w}, v) \mid \bar{w} \in C, v \in K e_{3}+K e_{4}, w \wedge v=0\right\}$ of degree -2 .

A line bundle $\mathcal{L}$ on $E$ of degree -2 induces a non constant morphism $E \rightarrow \mathbb{P}\left(H^{0}\left(E, \mathcal{L}^{*}\right)\right)$. This yields a bijection between the isomorphy classes of line bundles of degree 2 and the equivalence classes of non constant morphisms $E \rightarrow \mathbb{P}^{1}$ of degree 2 . Then $\mathcal{L}_{1}$ is not isomorphic to $\mathcal{L}_{2}$, since the two morphisms are not equivalent. The ruled surface $\tilde{S} \rightarrow E$ is equal to $\operatorname{Proj}\left(O_{E} \oplus \mathcal{L}\right)$, where $\mathcal{L}=\mathcal{L}_{1} \otimes \mathcal{L}_{2}^{-1}$ is any line bundle of degree 0 , not isomorphic to $O_{E}$. In particular, $\tilde{S}$ is not isomorphic to $\mathbb{P}^{1} \times E$.

3.2 The classification, using the singular locus

\subsection{1 $2^{2}$ does not occur as singular locus}

Lemma 3.1 The singular locus of a quartic ruled surface cannot be a conic.

Proof Suppose that the conic $D$, lying in a plane $H \subset \mathbb{P}(V)$, is the singular locus of some ruled quartic surface $S$, corresponding to a curve $C \subset \mathbb{P}\left(\Lambda^{2} V\right)$.

If $C$ has genus 1 , then $P(C)$ is contained in a tangent space of $G r$ at some point $\bar{w}_{0}$. The morphism $\bar{w} \in C \mapsto \bar{w} \cap \bar{w}_{0} \in \bar{w}_{0}$ has degree at least 2 and thus $\bar{w}_{0}$ belongs to the singular locus. This contradicts the assumption and implies that $C^{\text {norm }}$ has genus 0 . 
The morphism $f: C^{\text {norm }} \rightarrow D$, given by $\bar{w} \in C^{\text {norm }} \mapsto \bar{w} \cap H \in D$, has degree at most 2 , since the multiplicity of $D$ is 2 .

Suppose that the degree of $f$ is 1 . One can parametrize $C^{\text {norm }}$ with a parameter $t$ and choose coordinates for $\mathbb{P}(V)$ such that the line $\bar{w}(t) \in C^{\text {norm }}$ intersects the conic $D$ in the point $\left(0,1, t, t^{2}\right)$. Let $(1,0, a, b)$ with $a, b \in K(t)$ be another point of this line $\bar{w}(t)$. The Plücker coordinates of $\bar{w}(t)$ are

$$
p_{12}=1, p_{13}=t, p_{14}=t^{2}, p_{23}=-a, p_{24}=-b, p_{34}=t^{2} a-t b .
$$

Let $d$ be the common denominator of $a$ and $b$. Then $\left\{d p_{i j}\right\}$ are polynomials of degree $\leq 4$ and with $g . c . d .=1$. If $\alpha$ is a zero of $d$, then the line $\bar{w}(\alpha)$ lies in the plane $H$. Since this is not possible, $d=1$ and $a, b \in K[t]$. One obtains the contradiction that the line $\bar{w}(\infty)$ lies in the plane $H$.

Suppose that the degree of $f$ is 2 . One can parametrize $C^{\text {norm }}$ with parameter $t$, and choose coordinates for $\mathbb{P}(V)$ such that $\bar{w}(t) \mapsto\left(0,1, t^{2}, t^{4}\right) \in D$. The line $\bar{w}(t)$ goes through a point $(1,0, a, b)$ where $a, b \in K(t)$. The Plücker coordinates of $\bar{w}(t)$ are

$$
p_{12}=1, p_{13}=t^{2}, p_{14}=t^{4}, p_{23}=-a, p_{24}=-b, p_{34}=t^{4} a-t^{2} b .
$$

Let $d$ be the common denominator of $a$ and $b$. After multiplying the Plücker coordinates with $d$, the degrees are bounded by 4 . Hence $d=1$ and $a, b \in K[t]$. Further the degree of $a$ is $\leq 2$ and the degree of $c:=b-t^{2} a$ is $\leq 2$.

The symmetric polynomial $w(s) \wedge w(t)$ in $s, t$ can only have the factors $s+t$ and $s-t$. Indeed, $t \neq s$ and $w(s) \wedge w(t)=0$ implies that $\bar{w}(s) \cap \bar{w}(t) \in D$ and thus $s=-t$. It follows that $a=a_{0}+a_{2} t^{2}, c=c_{0}+c_{2} t^{2}$ and this contradicts that $C^{\text {norm }}$ is parametrized by $t$.

\subsubsection{Singular locus $1^{2}, 2^{2}$}

The curve $C^{\text {norm }}$, corresponding to a ruled quartic surface $S$ with this type of singular locus, has genus 0 by Observation 1.11. The singular locus is the union of a conic $D$ and a line $L$. The plane $H \supset D$ satisfies $S \cap H=C$. Thus $L$ does not lie in $H$ and the intersection $L \cap H$ is a point of $D$. As in the proof of Lemma 3.1, one shows that the morphism $C^{\text {norm }} \rightarrow D$, given by $\bar{w} \in C \mapsto \bar{w} \cap H \in D$, has degree 2 .

Let $D=\left\{\left(0,1, \mu^{2}, \mu\right) \mid \mu \in \mathbb{P}^{1}\right\}$ and $L=\left\{(1, \lambda, 0,0) \mid \lambda \in \mathbb{P}^{1}\right\}$. The equations for $D$ and $L$ are $t_{1}=t_{2} t_{3}-t_{4}^{2}=0$ and $t_{3}=t_{4}=0$. The equation $F$ for $S$ lies in the ideal $\left(t_{1}, t_{2} t_{3}-t_{4}^{2}\right)^{2} \cap\left(t_{3}, t_{4}\right)^{2}$. Thus $F=t_{1}^{2} A_{2}+t_{1}\left(t_{2} t_{3}-t_{4}^{2}\right) A_{1}+\left(t_{2} t_{3}-t_{4}^{2}\right)^{2}$ where $A_{2}$ and $A_{1}$ are homogeneous of degrees 2 and 1 . One may suppose that $A_{1}$ does not contain $t_{1}$. If $A_{1}$ contains $t_{2}$, then $F$ contains the monomial $t_{1} t_{2}^{2} t_{3}$ which is not possible. Hence $A_{1}$ is linear in $t_{3}, t_{4}$ and it follows that $A_{2}$ is homogeneous of degree 2 in $t_{3}, t_{4}$. Thus

$$
F=t_{1}^{2}\left(c_{1} t_{3}^{2}+c_{2} t_{3} t_{4}+c_{3} t_{4}^{2}\right)+t_{1}\left(t_{2} t_{3}-t_{4}^{2}\right)\left(c_{4} t_{3}+c_{5} t_{4}\right)+\left(t_{2} t_{3}-t_{4}^{2}\right)^{2} .
$$

We will show that an irreducible equation $F$ as above, defines a ruled surface. Consider a point $\left(0,1, \mu^{2}, \mu\right) \in D, \mu \neq 0, \infty$, then there is a $(1, \lambda, 0,0) \in L$ such that the line $\left\{\left(s, s \lambda+1, \mu^{2}, \mu\right) \mid s \in \mathbb{P}^{1}\right\}$ lies on the surface $F=0$. Indeed, substitution in $F$ yields the equation

$$
s^{2}\left(c_{1} \mu^{4}+c_{2} \mu^{3}+c_{3} \mu^{2}\right)+s^{2} \lambda \mu^{2}\left(c_{4} \mu^{2}+c_{5} \mu\right)+s^{2} \lambda^{2} \mu^{4}=0 .
$$

For general constants $c_{i}$ and general $\mu \neq 0, \infty$, this equations has two solutions for $\lambda$. If the equation has for every $\mu$ only one solution for $\lambda$, then one easily verifies that $F$ is reducible (in fact $F$ is then a square). 
Suppose now that $(1, \lambda, 0,0) \in L$ is given. The $\mu \neq 0, \infty$ such that the line $\{(s, s \lambda+$ $\left.\left.1, \mu^{2}, \mu\right) \mid s \in \mathbb{P}^{1}\right\}$ lies on $F=0$ are solutions of the equation

$$
\mu^{2}\left(\lambda^{2}+c_{4} \lambda+c_{1}\right)+\mu\left(c_{2}+\lambda c_{5}\right)+c_{3}=0 .
$$

(a) Suppose $c_{3} \neq 0$. If the equation has only one solution for $\mu \neq 0, \infty$, then $F$ is easily verified to be a square. The assumption that $F$ is irreducible, implies that there are for general $\lambda$ two solutions $\mu$. We conclude that the maps $C \rightarrow D$ and $C \rightarrow L$, given by $\bar{w} \in C \mapsto \bar{w} \cap H \in D$ and $\bar{w} \in C \mapsto \bar{w} \cap L \in L=\overline{e_{12}}$ both have degree 2. A further calculation shows that $P(C)=T_{G r, \overline{e_{12}}}, \overline{e_{12}} \notin C$ and the vector bundle $B$ has type $-2,-2$. There are still two cases, Number 11 and 12 .

(b) If $c_{3}=0$, then $c_{2}=c_{5}=0$ is excluded by $F$ is irreducible. Thus there is only one solution $\mu$. The maps $C \rightarrow D$ and $C \cdots \rightarrow L=\overline{e_{12}}$ have degrees 2 and 1 . Further calculation shows that $P(C)=T_{G r, \overline{e_{12}}}, \overline{e_{12}} \in C$ and the vector bundle $B$ has type $-1,-3$. This is Number 3 .

In Rohn's paper only case (a) is considered and this is treated as follows. The image $E$ of the morphism $C^{\text {norm }} \rightarrow D \times L$ is given by an irreducible bi-homogeneous form of bi-degree $(2,2)$. Since $C^{\text {norm }}$ has genus 0 , the curve $E$ has a singular point which is a node or a cusp. The embedding $E \subset D \times L \cong \mathbb{P}^{1} \times \mathbb{P}^{1}$ can be chosen to be symmetric if the field $K$ is algebraically closed. For $K=\mathbb{R}$ one can have a symmetric or an anti-symmetric embedding (see Sect. 4).

If $E$ has a node, then the equation, symmetric in $\lambda, \mu$, for the embedding is written as $A(\lambda, \mu):=a_{1} \lambda^{2} \mu^{2}+a_{2}\left(\mu^{2} \pm \lambda^{2}\right)+2 a_{3} \lambda \mu=0$, where $\lambda$ and $\mu$ are inhomogeneous coordinates for the rational curves $L$ and $D$. The \pm sign takes care of the real case where one also has to consider an anti-symmetric embedding. The singular point of $E$ corresponds to $\lambda=\mu=0$, which is the point $(0,1,0,0)$. The surface $S$ containing the family of the lines through the the pairs of points $\left\{(\lambda, 1,0,0),\left(0,1, \mu^{2}, \mu\right)\right\}$ satisfying $A(\lambda, \mu)=0, \lambda, \mu \neq 0$ has the equation

$$
a_{1} t_{1}^{2} t_{3}^{2}+a_{2}\left(\left(t_{2} t_{3}-t_{4}^{2}\right)^{2} \pm t_{1}^{2} t_{4}^{2}\right)+2 a_{3} t_{1} t_{4}\left(t_{2} t_{3}-t_{4}^{2}\right)=0 .
$$

There are various possibilities over $\mathbb{R}$ of the 'pinch points' on $L$ and $D$, i.e., the ramification points for the two projections $p r_{1}: E \rightarrow D, p r_{2}: E \rightarrow L$.

(i) All four are real if $\pm=+$ and $\frac{a_{3}^{2}-a_{2}^{2}}{a_{1} a_{2}}>0$.

Series XIII, no 8, corresponds to this case with additionally $a_{1}, a_{2}>0$.

(ii) No real ones, if $\pm=+$ and $\frac{a_{3}^{2}-a_{2}^{2}}{a_{1} a_{2}}<0$.

(iii) Real on $L$ and not real on $D$ if $\pm=-$ and $a_{1}>0, a_{2}<0$.

(iv) Not real on $D$ and real on $L$ if $\pm=-$ and $a_{1}>0, a_{2}>0$.

If $E$ has a cusp, then the equation, symmetric in $\lambda, \mu$, for the embedding $E \subset \mathbb{P}^{1} \times \mathbb{P}^{1}$ can be normalized (following Rohn) to

$$
\begin{aligned}
& (\lambda-\mu)^{2}-2 \lambda \mu(\lambda+\mu)+\lambda^{2} \mu^{2}=0 . \text { This leads to the equation } \\
& t_{1}^{2} t_{3}^{2}-2 t_{1} t_{3}\left(t_{1} t_{4}+t_{2} t_{3}-t_{4}^{2}\right)+\left(t_{1} t_{4}-t_{2} t_{3}+t_{4}^{2}\right)^{2}=0 \text { for } S .
\end{aligned}
$$

\subsubsection{Singular locus $1^{3}$}

The line with multiplicity 3 is chosen to be $t_{3}=t_{4}=0$. Then the equations have the form $t_{1} A_{1}+t_{2} A_{2}+A_{3}=0$ with $A_{1}, A_{2}, A_{3}$ homogeneous in $t_{3}, t_{4}$ of degree $3,3,4$; 
g.c.d. $\left(A_{1}, A_{2}, A_{3}\right)=1$ and $A_{1}, A_{2}$ are linearly independent. Conversely, one easily verifies that the above equation defines a ruled surface of degree 4 .

The pair $\left(A_{1}, A_{2}\right)$ is unique up to taking linear combinations (and linear changes of $\left.t_{3}, t_{4}\right)$. In other words the morphism $f: \mathbb{P}^{1} \rightarrow \mathbb{P}^{1}$, given by $\left(t_{3}: t_{4}\right) \mapsto\left(A_{1}: A_{2}\right)$, is unique and can have degree 3, 2 and 1 . In the first case there are three possibilities for $f$ (see the beginning of $\S 3.1 .1)$. In the second case one can normalize $\left(A_{1}, A_{2}\right)=d\left(t_{3}, t_{4}\right) \cdot\left(t_{3}^{2}, t_{4}^{2}\right)$ and in the third case $\left(A_{1}, A_{2}\right)=d\left(t_{3}, t_{4}\right) \cdot\left(t_{3}, t_{4}\right)$.

The term $A_{3}$ can be changed into $A_{3}+\ell_{1} A_{1}+\ell_{2} A_{2}$ with $\ell_{1}, \ell_{2}$ homogeneous in $t_{3}, t_{4}$ of degrees 1 , by replacing $t_{1}, t_{2}$ by $t_{1}+\ell_{1}, t_{2}+\ell_{2}$.

1. g.c.d. $\left(A_{1}, A_{2}\right)=1$ and $A_{3}=0$. Number $1 a, b, c$.

2. g.c.d. $\left(A_{1}, A_{2}\right)=1$ and $A_{3} \notin\left\{\ell_{1} A_{1}+\ell_{2} A_{2}\right\}$. Number $13 a, b, c$, XIII 6.

3. g.c.d. $\left(A_{1}, A_{2}\right)$ has degree 1 . Number 8,9 .

4. g.c.d. $\left(A_{1}, A_{2}\right)$ has degree 2 and is not a square. Then $\overline{e_{12}}$ is a node. Number 4 , XIII 7.

5. g.c.d. $\left(A_{1}, A_{2}\right)$ has degree 2 and is a square. $\overline{e_{12}}$ is a cusp. Number 5 .

\subsubsection{Singular locus $3^{2}$}

The twisted cubic curve $T C:=\left\{\left(1, \lambda, \lambda^{2}, \lambda^{3}\right) \mid \lambda \in \mathbb{P}^{1}\right\}$ is the singular locus of $S$. The homogeneous ideal of $T C$ is generated by the three homogeneous forms $X=t_{1} t_{3}-t_{2}^{2}, Y=$ $t_{2} t_{3}-t_{1} t_{4}, Z=t_{2} t_{4}-t_{3}^{2}$. There are two relations $t_{3} X+t_{2} Y+t_{1} Z=t_{4} X+t_{3} Y+t_{2} Z=0$. The equation $F$ of $S$ is homogeneous of degree 4 and lies in the ideal $(X, Y, Z)^{2} \subset K\left[t_{1}, t_{2}, t_{3}, t_{4}\right]$. A computation in the ring $R:=K\left[\frac{t_{2}}{t_{1}}, \frac{t_{3}}{t_{1}}, \frac{t_{4}}{t_{1}}\right]$ shows that the element $G:=F\left(1, \frac{t_{2}}{t_{1}}, \frac{t_{3}}{t_{1}}, \frac{t_{4}}{t_{1}}\right)$ of total degree $\leq 4$, lying in the ideal $\left(R \frac{X}{t_{1}^{2}}+R \frac{Y}{t_{1}^{2}}\right)^{2} \subset R$, is a homogeneous polynomial in the terms $\frac{X}{t_{1}^{2}}, \frac{Y}{t_{1}^{2}}, \frac{Z}{t_{1}^{2}}$ of degree 2. It follows that $F\left(t_{1}, t_{2}, t_{3}, t_{4}\right)=H(X, Y, Z)$, where $H$ is a homogeneous form of degree 2 .

Consider the morphism $f: \mathbb{P}(V) \backslash T C \rightarrow \mathbb{P}^{2}$, given by $\left(t_{1}, t_{2}, t_{3}, t_{4}\right) \mapsto(X, Y, Z)$. The fibres of $f$ are the lines of $\mathbb{P}(V)$ intersecting $T C$ with total multiplicity 2 . Thus a fibre is a corde of $T C$ or a tangent line of $T C$. Let $H(X, Y, Z)$ be homogeneous of degree 2 . Then the closure of the preimage under $f$ of the subscheme $H=0$ of $\mathbb{P}^{2}$ is the ruled surface $S$ given by the equation $F\left(t_{1}, t_{2}, t_{3}, t_{4}\right):=H(X, Y, Z)$. Further $F$ is irreducible and reduced if and only if $H=0$ is a conic. In the sequel we suppose that $\{H=0\}$ is a conic and we classify the possibilities.

The surface with $H=T:=Y^{2}-4 X Z$ is rather special. It consists of all tangent lines of $T C$ (see Corollary 1.7). For any other conic $H=0$, the intersection with $T=0$ has multiplicity 4. In the general case, the intersection of the two conics consists of 4 points. This is Series XIII 9, 10.

Suppose that the intersection has at least one point with multiplicity $>1$. The projective space $\mathbb{P}^{3}$ admits an automorphism which preserves the curve $T=Y^{2}-4 X Z=0$ and brings this point to $(X, Y, Z)=(0,0,1)$. Then $H$ has the form $X Z+a X^{2}+b X Y+c Y^{2}$. One has the following cases for the intersection.

(i) $a X^{2}+b X Y+(c+1 / 4) Y^{2}=0$ has two distinct solutions (i.e., $\left.b^{2}-a(4 c+1) \neq 0\right)$ and $(c+1 / 4) \neq 0$. Then the intersection consists of one point with multiplicity 2 and two points with multiplicity 1 .

(ii) $a X^{2}+b X Y+(c+1 / 4) Y^{2}=0$ has two distinct solutions and $(c+1 / 4)=0$. Then the intersection consists of one point with multiplicity 3 and one point with multiplicity 1 .

(iii) $a X^{2}+b X Y+(c+1 / 4) Y^{2}=0$ has one solution (i.e. $\left.b^{2}-a(4 c+1)=0\right)$ and $(c+1 / 4) \neq 0$. Then the intersection consists of two points with multiplicity 2 . 
(iv) $a X^{2}+b X Y+(c+1 / 4) Y^{2}=0$ has one solution (i.e. $\left.b^{2}-a(4 c+1)=0\right),(c+1 / 4)=0$ and $a \neq 0$. Then the intersection consists of one point with multiplicity 4 .

Thus we found in total six distinct cases for $3^{2}$ (compare [2]). As we will show below, there is a further natural subdivision of these classes.

Let $C \subset G r$ be the curve associated to the surface $S_{H}$ associated to the irreducible $H=H(X, Y, Z)$ of degree 2. The morphism $C \rightarrow\{H=0\} \subset \mathbb{P}^{3}$ is clearly an isomorphism. Thus $C$ is a non singular rational curve. It is clear that $P(C)$ does not lie in two tangent spaces of $G r$. Moreover, since $C$ is not singular, one must have $\operatorname{dim} P(C)=4$. For the surfaces $S_{T}$ and $S_{H}$ with $H=X Z+a X^{2}-\frac{1}{4} Y^{2}$ with $a \neq 0$ (case (iv) above), $P(C)$ is not a tangent space. For the remaining 4 classes there are, a priori, now two possibilities:

(a) If $P(C)$ is not a tangent space, then $B$ has type $-2,-2$. There are in total six cases and they fill up Number 14.

(b) If $P(C)$ is a tangent space $T_{G r}, \bar{w}_{0}$, then $B$ has type $-1,-3$. Number $2 a, b, c$. These cases are explained as follows.

The line $\bar{w}_{0}$ coincides with $\overline{e_{12}}$ of the cases $2 a, b, c$. The image $f\left(\bar{w}_{0}\right)$ is the conic given by $H=0$. The possibilities for intersection of $H=0$ with $T=0$ reflect the possibilities for the ramification of the degree 3 morphism in $2 a, b, c$. Case $2 a$ corresponds to (iii) above; case $2 b$ to (i) above; case $2 c$ to the case where the intersection consists of 4 points.

\subsubsection{Singular locus $1^{2}$}

From Observations 1.5 and $\S 1.4$, one obtains that the genus of $C$ is 1 . Further $P(C)$ lies in only one tangent space of $G r$, say at the point $\overline{e_{12}}$, since otherwise the surface $S$ has two skew singular lines. The morphism $C \rightarrow \overline{e_{12}}$, given by $\bar{w} \in C \mapsto \bar{w} \cap \overline{e_{12}}$, has degree 2 since the singular line $\overline{e_{12}}$ has multiplicity 2 . This map has 4 ramification points and we obtain for, say, $t \neq 0,1, \lambda, \infty$ two lines of $C$ through the point $(1, t, 0,0) \in \overline{e_{12}}$. The map $t \mapsto P(t)$, where $P(t) \supset \overline{e_{12}}$ denotes the plane through these two lines (or one line with multiplicity 2 ), has degree 1 . We may suppose that $P(t) \cap\{(0,0, *, *)\}=(0,0,1, t)$. Since $\overline{e_{12}}$ has multiplicity 2 , the equation for $S$ has the form

$$
t_{1}^{2} A+t_{2}^{2} B+t_{1} t_{2} C+t_{1} D+t_{2} E+F ; A, B, C, D, E, F \text { homogeneous in } t_{3}, t_{4} .
$$

For any point $\left(a_{1}, a_{2}, 0,0\right) \in \overline{e_{12}}$, the plane $a_{2} t_{3}-a_{1} t_{4}=0$ meets $S$ in $\overline{e_{12}}$ and two lines (or one with multiplicity 2$)$ through $\left(a_{1}, a_{2}, 0,0\right)$. This implies that $t_{1}^{2} A\left(t_{3}, t_{4}\right)+t_{2}^{2} B\left(t_{3}, t_{4}\right)+$ $t_{1} t_{2} C\left(t_{3}, t_{4}\right)$ is a multiple of $\left(t_{4} t_{1}-t_{3} t_{2}\right)^{2}$ and that $t_{1} D\left(t_{3}, t_{4}\right)+t_{2} E\left(t_{3}, t_{4}\right)$ is divisible by $\left(t_{4} t_{1}-t_{3} t_{2}\right)$. After changing the variables $t_{1}, t_{2}$ we are reduced to two possible equations for $S$ :

$$
\left(t_{4} t_{1}-t_{3} t_{2}\right)^{2}+H\left(t_{3}, t_{4}\right)=0 \text { and } G\left(t_{3}, t_{4}\right)\left(t_{4} t_{1}-t_{3} t_{2}\right)+H\left(t_{3}, t_{4}\right)=0 .
$$

The line $\overline{e_{12}}$ has multiplicity 3 for the second equation. Thus only the first equation is possible with $H$ not a square since $S$ is irreducible. Moreover, the ruled surface defined by this equation has $\overline{e_{12}}$ as singular locus if and only if $H$ has no multiple factor. This is Number 15 .

Rohn found an equation $a\left(t_{3}^{2} \pm t_{4}^{2}\right)+2 b t_{3}^{2} t_{4}^{2}+c\left(t_{4} t_{2}-t_{4} t_{1}\right)^{2}=0$ of this form. The sign \pm distinguishes two classes of real cases. For $\pm=+$ and $\frac{b}{a}<-1$, the four ramification points of $C \rightarrow \overline{e_{12}}$ are real. This is XIII, no 5 .

Remark The equation $\left(t_{4} t_{1}-t_{3} t_{2}\right)^{2}+H\left(t_{3}, t_{4}\right)=0$ where $H$ has no multiple factors, is valid for any field $K$. If $K$ is algebraically closed, then $H$ is determined by the $j$-invariant of the four zeros of $H$ in $\mathbb{P}^{1}$. For a general field $K$ there are forms for $H$. 


\subsubsection{Singular locus $1^{2}, 1^{2}$, int, i.e., intersecting lines}

The two intersecting lines $L_{1}, L_{2}$, making up the singular locus of the ruled quartic surface $S$, lie in a plane $H$. For $\bar{w} \in C$ and $\bar{w} \neq L_{1}, L_{2}$ the intersection $\bar{w} \cap H$ is a point of $L_{1} \cup L_{2}$. The induced morphism $C^{\text {norm }} \rightarrow L_{1} \cup L_{2}$ has, say, the line $L_{1}$ as image. Thus we find a non constant morphism $f: C^{\text {norm }} \rightarrow L_{1}$ and $P(C)$ lies in the tangent space of $G r$ at the point $L_{1}$. For $q \in L_{2}$ and $q \notin L_{1}$, there is no $\bar{w} \in C, \bar{w} \neq L_{1}, L_{2}$ with $q \in \bar{w}$. One concludes that $L_{2} \in C$. Moreover $L_{2}$ is a singular point $s$ of $C$ since $L_{2}$ belongs to the singular locus. In particular, $C$ is a rational curve and $\operatorname{dim} P(C)=3$.

If $P(C)$ lies in the tangent space of $G r$ at another point $M \in G r$, then one obtains a morphism $C \rightarrow M$ by $\bar{w} \mapsto \bar{w} \cap M$. Since $C$ has a singular point, this morphism has degree $>1$ and one finds the contradiction that $M$ belongs to the singular locus. Thus $P(C)$ lies in a single tangent space.

The rational map $C \cdots \rightarrow L_{1}$, given by $\bar{w} \mapsto \bar{w} \cap L_{1}$, is well defined at the singular point $s \in C$. Then $f$ has degree $>1$ and its degree is 2 , since $L_{1}$ has multiplicity 2 . Further $L_{1} \notin C$, otherwise the multiplicity of $L_{1}$ would be 3 .

For a suitable basis of $V$ and parametrization of $C^{\text {norm }}$, the morphism $C^{\text {norm }} \rightarrow L_{1}$ has the form $\bar{w}(t) \mapsto\left(1, t^{2}, 0,0\right)$. Let $b:=\left(b_{1}, b_{2}, b_{3}, b_{4}\right)$, with all $b_{i} \in K[t]$ and g.c.d. $\left(b_{1}, \ldots, b_{4}\right)=1$, be another point of the line $\bar{w}(t)$. By subtracting a multiple of $\left(1, t^{2}, 0,0\right)$ one arrives at $\operatorname{deg} b_{2} \leq 1$.

The Plücker coordinates of $\bar{w}(t)$ are $\left(b_{2}-t^{2} b_{1}, b_{3}, b_{4}, t^{2} b_{3}, t^{2} b_{4}, 0\right)$ and thus deg $b_{1}$, deg $b_{3}, \operatorname{deg} b_{4} \leq 2$. The morphism $C \rightarrow \mathbb{P}^{1} ; \bar{w}(t) \mapsto\left(b_{3}(t): b_{4}(t)\right)$, is well defined and not constant. Since $C$ is singular, this morphism has degree 2 . The corresponding morphism $g: C^{\text {norm }} \rightarrow \mathbb{P}^{1}$ of degree 2 factors over $C$. If the singular point of $C$ is a cusp for $t=\infty$, then $t=\infty$ is a ramification point and $g$ has the form $g(t)=\left(1:(t+\alpha)^{2}\right)$. If the singular point of $C$ is a node, corresponding to $t= \pm 1$, then $g(t)=\left(1:\left(\frac{a t+b}{c t+d}\right)^{2}\right)$ satisfies $g(1)=g(-1)$ and hence $g(t)=\left(1:\left(\frac{t+\beta}{\beta t+1}\right)^{2}\right)$ with $\beta^{2} \neq 1$.

Suppose that $C$ has a cusp, then $\left(b_{3}(t), b_{4}(t)\right)=\left(1,(t+\alpha)^{2}\right)$ and $b_{1}, b_{2}$ can be normalized to constant multiples of $t$. The condition that $t=\infty$ is a cusp for $C$ implies $b_{1}=0$ and so we arrive at $b=\left(0, t, 1,(t+\alpha)^{2}\right)$. The equation reads

$$
\left(t_{2} t_{3}-t_{1} t_{4}-\alpha^{2} t_{1} t_{3}+\alpha t_{3}^{2}\right)^{2}-t_{3} t_{4}\left(2 \alpha t_{1}-t_{3}\right)^{2}=0 . \text { Number } 10 \mathrm{~b} .
$$

Suppose that $C$ has a node, then $\left(b_{3}, b_{4}\right)=\left((\beta t+1)^{2},(t+\beta)^{2}\right)$ with $\beta^{2} \neq 1$. For $\beta=0$, one can normalize $b_{1}, b_{2}$ to constant multiples of $t$. The condition $\bar{w}(1)=\bar{w}(-1)$ implies that $b_{1}=b_{2}=c t \neq 0$. The equation reads

$$
c^{2} t_{3} t_{4}\left(t_{3}-t_{4}\right)^{2}-\left(t_{1} t_{4}-t_{2} t_{3}\right)^{2}=0 . \text { Number } 10 a .
$$

For $\beta^{2} \neq 0,1$, one can normalize $b_{1}, b_{2}$ to constants and the condition $\bar{w}(1)=\bar{w}(-1)$ implies $b_{1}=b_{2}=c \neq 0$. The equation reads

$$
t_{3} t_{4}\left\{2 \beta\left(t_{1}-t_{2}\right)+c\left(1-\beta^{2}\right)\left(t_{3}-t_{4}\right)\right\}^{2}-\left\{-t_{1}\left(\beta^{2} t_{3}+t_{4}\right)+t_{2}\left(t_{3}+\beta^{2} t_{4}\right)\right\}^{2}=0 .
$$

Again Number $10 a$.

Rohn found the two similar equations

$$
a t_{3} t_{4}^{3}+\left(t_{1} t_{4}-t_{2} t_{3}\right)^{2}=0 \text { and } a t_{4}^{4}+2 b t_{3}^{2} t_{4}^{2}+\left(t_{1} t_{4}-t_{2} t_{3}\right)^{2}=0 .
$$




\subsubsection{Singular locus $1^{2}, 1^{2}$, skew lines}

The skew lines can be supposed to be $\overline{e_{12}}, \overline{e_{34}}$. Every monomial in the equation $F$ of $S$ is divisible by one of the terms $t_{1}^{2}, t_{1} t_{2}, t_{2}^{2}$ and by one of the terms $t_{3}^{2}, t_{3} t_{4}, t_{4}^{2}$. Therefore $F$ is bihomogeneous of degree $(2,2)$ and $F$ defines a Zariski closed subset of $\overline{e_{12}} \times \overline{e_{34}} \cong \mathbb{P}^{1} \times \mathbb{P}^{1}$, which is an irreducible curve $E$. One considers the morphism $f: \mathbb{P}(V) \backslash \overline{e_{12}} \cup \overline{e_{34}} \rightarrow \mathbb{P}^{1} \times \mathbb{P}^{1}$, given by $\left(a_{1}, a_{2}, a_{3}, a_{4}\right) \mapsto\left(\left(a_{1}, a_{2}\right),\left(a_{3}, a_{4}\right)\right)$. Then $S$ is the Zariski closure of $f^{-1}(E)$. The curve $E$ has no singularities since otherwise the singular locus of $S$ would contain another line. Thus $E$ is a curve of genus 1 . One easily sees that $C$ identifies with $E$ and that $P(C)$ lies in the two tangent space of $G r$ at the points $\overline{e_{12}}$ and $\overline{e_{34}}$. Number 16.

In the above the bases of the two vector spaces $K e_{1}+K e_{2}$ and $K e_{3}+K e_{4}$ (or equivalently the parametrization of $\overline{e_{12}}$ and $\overline{e_{34}}$ ) can be chosen in a suitable way. Rohn (see Sect. 4) shows that for $K=\mathbb{C}$ these bases can be chosen such that the equation $F$ becomes symmetric, i.e., $F\left(t_{1}, t_{2}, t_{3}, t_{4}\right)=F\left(t_{3}, t_{4}, t_{1}, t_{2}\right)$. For $K=\mathbb{R}$ the results of Rohn are more complicated. These results are essential for the understanding of the models in Series XIII, 1,2,3,4 of quartic ruled surfaces with two skew lines of singularities.

\subsubsection{Singular locus $1^{2}, 1^{2}, 1^{2}$}

Let $L_{1}, L_{2}, L_{3}$ denote the singular lines with multiplicity 2 . Suppose that the lines $L_{1}, L_{2}$ are skew. We may suppose $L_{1}=\overline{e_{12}}, L_{2}=\overline{e_{34}}$. From Lemma 1.10 it follows that the equation $F$ of the surface $S$ is bihomogeneous of degree $(2,2)$ in the pairs of variables $t_{1}, t_{2}$ and $t_{3}, t_{4}$. The curve $E \subset \overline{e_{12}} \times \overline{e_{34}} \cong \mathbb{P}^{1} \times \mathbb{P}^{1}$, defined by $F$, has one singular point corresponding to the line $L_{3}$. This point is a node or a cusp. Number 6, 7. Not in Series XIII.

The parametrization of $\overline{e_{12}}$ and $\overline{e_{34}}$ can be chosen (see Sect. 4) in order to obtain the standard equations of Rohn

$$
a_{1} \lambda^{2} \mu^{2}+a_{2}\left(\lambda^{2} \pm \mu^{2}\right)+2 a_{3} \lambda \mu=0 \text { and } \lambda^{2} \mu^{2}+(\lambda-\mu)^{2}-2 \lambda \mu(\lambda+\mu)=0,
$$

where $\lambda=\frac{t_{2}}{t_{1}}, \mu=\frac{t_{4}}{t_{3}}$.

The next case to consider is $L_{1} \cap L_{2}, L_{1} \cap L_{3}, L_{2} \cap L_{3} \neq \emptyset$. The three lines cannot lie in a plane $H$ since otherwise the curve $H \cap S$ has degree 6. It follows that $L_{1} \cap L_{2} \cap L_{3}$ is one point. We may suppose that $L_{1}$ is given by $t_{1}=t_{2}=0, L_{2}$ by $t_{1}=t_{3}=0$ and $L_{3}$ by $t_{2}=t_{3}=0$. Every monomial of the equation $F$ is divisible by $t_{1}^{a_{0}} t_{2}^{a_{1}}$ with $a_{0}+a_{1}=2$, by $t_{1}^{b_{0}} t_{3}^{b_{1}}$ with $b_{0}+b_{1}=2$ and by $t_{2}^{c_{0}} t_{3}^{c_{1}}$ with $c_{0}+c_{1}=2$. The $t_{4}$-part of $F$ can only be $c \cdot t_{1} t_{2} t_{3} t_{4}$. If $c=0$, then $F$ defines a cone. Otherwise one can reduce to the equation $\left(t_{2}^{2} t_{3}^{2}+t_{1}^{2} t_{3}^{2}+t_{1}^{2} t_{2}^{2}\right)+t_{4} t_{1} t_{1} t_{3}=0$ (or equivalently $\left(t_{2} t_{3}+t_{1} t_{3}+t_{1} t_{2}\right)^{2}+t_{4} t_{1} t_{2} t_{3}=0$ ). This equation defines the Steiner's Roman surface and the three singular lines are in fact the only lines on this surface.

\section{Rohn's symmetric form for bi-degree $(2,2)$}

$\mathrm{K}$. Rohn proves that over the field $K=\mathbb{C}$, there is an identification of $\overline{e_{12}} \times \overline{e_{34}}$ with $\mathbb{P}^{1} \times \mathbb{P}^{1}$ such that the equation $F$ of bi-degree $(2,2)$ is symmetric in the pairs of variables $t_{1}, t_{2}$ and $t_{3}, t_{4}$, i.e., $F\left(t_{3}, t_{4}, t_{1}, t_{2}\right)=F\left(t_{1}, t_{2}, t_{3}, t_{4}\right)$. This leads to only a few standard forms for $F$.

Over the field $\mathbb{R}$, there are more possibilities. First of all, $\overline{e_{12}}, \overline{e_{34}}$ can be a pair of conjugated lines over $\mathbb{C}$. Secondly, even if $\overline{e_{12}}, \overline{e_{34}}$ are real lines, then the above identification need not be defined over $\mathbb{R}$. Thirdly, there are various possibilities over $\mathbb{R}$ for the ramification 
points of the two projections $C \rightarrow \mathbb{P}^{1}$. The models Series XIII, nr. 1,2,3,4 represent some of these cases. A 'modern version' of this work of Rohn is as follows.

Consider the closed subset $E$ of $\mathbb{P}^{1} \times \mathbb{P}^{1}$, defined by a bi-homogeneous form $F$ of bi-degree $(2,2)$. To start we consider the case that $F$ is irreducible and $E$ is non singular and thus $E$ has genus 1 . We call the embedding $E \subset \mathbb{P}^{1} \times \mathbb{P}^{1}$ symmetric if $(p, q) \in E \Rightarrow(q, p) \in E$.

Theorem 4.1 [K. Rohn] For a given embedding $E \subset \mathbb{P}^{1} \times \mathbb{P}^{1}$ as above, there exists an automorphism $f$ of the first factor, such that the new embedding $E \subset \mathbb{P}^{1} \times \mathbb{P}^{1} \stackrel{f \times 1}{\rightarrow} \mathbb{P}^{1} \times \mathbb{P}^{1}$ is symmetric.

Proof The required automorphism $f$ of $\mathbb{P}^{1}$ has the property $(p, q) \in E \Rightarrow\left(f^{-1} q, f p\right) \in E$. In particular, the morphism $C:(p, q) \mapsto\left(f^{-1} q, f p\right)$ is an automorphism of $E$ of order 2 . We assume that $f$ exists, try to find its explicit form and then use this form to produce an $f$ with the required property.

Some explicit information concerning the automorphisms of order 2 of $E$ is needed. For this purpose, we choose a point $e_{0} \in E$. This makes $E$ into an elliptic curve (and the addition of two points $a, b$ is written as $a+b$ ). Consider the automorphisms $\sigma$ and $\tau_{a}$ (any $a \in E$ ), given by $\sigma(p)=-p$ and $\tau_{a}(p)=p+a$. One verifies that the automorphisms of order 2 of $E$ are:

(a) $\sigma \tau_{a}$ for any point $a$ on $E$,

(b) $\tau_{a}$ where $a \neq 0$ is a point of order two on $E$.

Division of $E$ by the action of an element in the first class yields $\mathbb{P}^{1}$ and division by the action of an element in the second class yields an elliptic curve. Thus the two projections $p r_{i}: E \rightarrow \mathbb{P}^{1}$ correspond to distinct elements $\sigma \tau_{a_{1}}$ and $\sigma \tau_{a_{2}}$ of order 2 with the property $p r_{i} \circ \sigma \tau_{a_{i}}=p r_{i}$ for $i=1,2$.

The assumption on $f$ and the definition of $C$ are equivalent to $\operatorname{pr}_{2}(C e)=f\left(p r_{1}(e)\right)$ for any $e \in E$. Replacing $e$ by $\sigma \tau_{a_{1}} e$ does not change the right hand side. Thus $C \sigma \tau_{a_{1}} e$ is either $C e$ or $\sigma \tau_{a_{2}} C e$. The first equality can only hold for four elements $e \in E$. Hence the second equality holds for almost all $e$ and thus holds for all $e$. We conclude that $C \sigma \tau_{a_{1}}=\sigma \tau_{a_{2}} C$.

Suppose that $C=\sigma \tau_{c}$. The equality $\sigma \tau_{c} \sigma \tau_{a_{1}}=\sigma \tau_{a_{2}} \sigma \tau_{c}$ is equivalent to $2 c=a_{1}+a_{2}$. There are 4 solutions $c$ of this equation.

Suppose that $C=\tau_{c}$ with $c$ an element of order 2. Then one finds the contradiction $a_{1}=a_{2}$.

Take $C=\sigma \tau_{c}$ for some $c$ with $2 c=a_{1}+a_{2}$. Define $f$ by the formula $f\left(p r_{1}(e)\right):=$ $p r_{2}(C e)$. This is well defined because of $C \sigma \tau_{a_{1}}=\sigma \tau_{a_{2}} C$. It is easily verified that $f$ is an isomorphism and has the required property.

Let $E \subset \mathbb{P}^{1} \times \mathbb{P}^{1}$ be a symmetric embedding and the homogeneous coordinates of the two projective lines are denoted by $x_{0}, x_{1}$ and $y_{0}, y_{1}$. Let $\left\{p_{1}, p_{2}, p_{3}, p_{4}\right\} \subset \mathbb{P}^{1}$ denote the four ramification points of the projection $p r_{1}: E \rightarrow \mathbb{P}^{1}$. There is an automorphism $s$ of order two which permutes each of the pairs $\left\{p_{1}, p_{2}\right\}$ and $\left\{p_{3}, p_{4}\right\}$. The two fixed points of $s$ can be supposed to be $0, \infty$ and thus $s$ has the form $s\left(x_{0}, x_{1}\right)=\left(x_{0},-x_{1}\right)$. The four ramification points are then $\{(1, \pm d)\}$ and $\{(1, \pm e)\}$. By scaling $\left(x_{0}, x_{1}\right) \mapsto\left(x_{0}, \lambda x_{1}\right)$ with $\lambda^{2} e d= \pm 1$ we arrive at four ramification points $\left\{\left(1, \pm d^{ \pm 1}\right\}\right.$ (with of course $d^{4} \neq 1$ ). Write $F=A y_{0}^{2}+B y_{0} y_{1}+C y_{1}^{2}$. Then the four ramification points of $p r_{1}$ are the zeros of the discriminant $B^{2}-4 A C$ and thus $B^{2}-4 A C=x_{1}^{4}+b x_{1}^{2} x_{0}^{2}+x_{0}^{4}$ with $b=-\left(d^{2}+d^{-2}\right)$. Then we obtain the normal form of $K$. Rohn for $F$ :

$$
a_{1}\left(x_{0}^{2} y_{0}^{2}+x_{1}^{2} y_{1}^{2}\right)+a_{2}\left(x_{0}^{2} y_{1}^{2}+x_{1}^{2} y_{0}^{2}\right)+2 a_{3} x_{0} x_{1} y_{0} y_{1} \quad \text { with } a_{1} a_{2} \neq 0
$$


or in Rohn's notation $a_{1}\left(\lambda^{2} \mu^{2}+1\right)+a_{2}\left(\lambda^{2}+\mu^{2}\right)+2 a_{3} \lambda \mu$ with $\lambda=\frac{x_{1}}{x_{0}}, \mu=\frac{y_{1}}{y_{0}}$, and with discriminant $x_{1}^{4}+b x_{0}^{2} x_{1}^{2}+x_{0}^{4}$ and $b=\frac{a_{1}^{2}+a_{2}^{2}-a_{3}^{2}}{a_{1} a_{2}} \neq \pm 2$.

The above calculations are valid over any algebraically closed field of characteristic $\neq 2$. Now we analyze the more complicated situation over the field $\mathbb{R}$. Assume that the two lines $\overline{e_{12}}, \overline{e_{34}}$ and $E$ are defined over $\mathbb{R}$. Assume moreover that $E(\mathbb{R})$ is not empty (indeed otherwise the real model for the corresponding surface has no points). Fix a real point $e_{0}$ as the neutral element of $E$. The group $E(\mathbb{R})$ is either isomorphic to the circle $\mathbb{R} / \mathbb{Z}$ (the connected case) or to $\mathbb{R} / \mathbb{Z} \times \mathbb{Z} / 2 \mathbb{Z}$ (the disconnected case). In the connected case $E(\mathbb{R})$ has two elements of order dividing 2 and in the disconnected case there are 4 such elements. The collection of the real automorphisms of order two of $E$ consists of the $\sigma \tau_{a}$ with $a \in E(\mathbb{R})$ and $\tau_{v}$ where $v$ is a real point of order 2. Now we revisit the proof of the theorem for the case $K=\mathbb{R}$.

The connected case. The fixed points $b$ of $\sigma \tau_{a_{1}}$ (note that $a_{1} \in E(\mathbb{R})$ ) are the solutions of $2 b=-a_{1}$. Two of the $b$ 's are real. The other two are complex conjugated. Hence two of the ramification points for $p r_{1}: E \rightarrow \mathbb{P}^{1}$ are real, the other two are complex conjugated. The same holds for the ramification points of $p r_{2}: E \rightarrow \mathbb{P}^{1}$.

For the element $c$ with $2 c=a_{1}+a_{2}$ there are two real choices. Thus the real version of the theorem remains valid in this case. Two of the four ramification points are real and the other two are complex conjugated. One can normalize such that the ramification points are $\pm d, \pm i d^{-1}$ and this leads to Rohn's normal equation

$$
a_{1}\left(-x_{0}^{2} y_{0}^{2}+x_{1}^{2} y_{1}^{2}\right)+a_{2}\left(x_{0}^{2} y_{1}^{2}+x_{1}^{2} y_{0}^{2}\right)+2 a_{3} x_{0} x_{1} y_{0} y_{1} \text { with real } a_{1}, a_{2}, a_{3} .
$$

The disconnected case. There are 4 real fixed points of $\sigma \tau_{a_{1}}$ if $a_{1}$ lies in the component of the identity of $E(\mathbb{R})$. In the opposite case there are no real solutions of $2 b=a_{1}$, The same holds for $\sigma \tau_{a_{2}}$ and for the solutions of the equation $2 c=a_{1}+a_{2}$. Hence there are cases where no real automorphism $f$ exist. All cases can be listed by:

(a) 4 real ramification points for $p r_{1}$ and for $p r_{2}$ and 4 real solutions for $c$,

(b) no real ramification points for $p r_{1}$ and $p r_{2}$ and 4 real solutions for $c$,

(c) 4 real ramification points for $p r_{1}$, none for $p r_{2}$ and no real solution for $c$,

(d) 4 real ramification points for $p r_{2}$, none for $p r_{1}$ and no real solution for $c$.

Suppose that $c$ can be chosen to be real. For Rohn's normal form one needs an automorphism $s$ permuting each pair $\left\{p_{1}, p_{2}\right\}$ and $\left\{p_{3}, p_{4}\right\}$. One may suppose that the each pair is invariant under complex conjugation. Then the resulting $s$ is also real.

For the cases (a) and (b) the standard equation is indeed

$$
\begin{aligned}
& a_{1}\left(x_{0}^{2} y_{0}^{2}+x_{1}^{2} y_{1}^{2}\right)+a_{2}\left(x_{0}^{2} y_{1}^{2}+x_{1}^{2} y_{0}^{2}\right)+2 a_{3} x_{0} x_{1} y_{0} y_{1} \\
& \text { and discriminant } x_{1}^{4}+\frac{a_{1}^{2}+a_{2}^{2}-a_{3}^{2}}{a_{1} a_{2}} x_{1}^{2} x_{0}^{2}+x_{0}^{4} .
\end{aligned}
$$

with $a_{1}, a_{2}, a_{3} \in \mathbb{R}$. One easily calculates that $\frac{\left(a_{1}+a_{2}\right)^{2}-a_{3}^{2}}{a_{1} a_{2}}<0$ corresponds to (a) and $\frac{\left(a_{1}+a_{2}\right)^{2}-a_{3}^{2}}{a_{1} a_{2}}>0$ corresponds to (b).

For the cases (c) and (d) there is no real symmetric normal form for $F$. In case (c) (case (d) is similar), Rohn's real normal form we will call anti-symmetric, because of its form

$$
a_{1}\left(x_{0}^{2} y_{0}^{2}-x_{1}^{2} y_{1}^{2}\right)-a_{2}\left(x_{0}^{2} y_{1}^{2}-x_{1}^{2} y_{0}^{2}\right)+2 a_{3} x_{0} x_{1} y_{0} y_{1} .
$$

The models 1,2 and 3 of Series XIII deal with a pair of real skew double lines. In the terminology of Rohn, a pinch point is a ramification point for one of the two projections 
$p r_{1}, p r_{2}$ and situated on $L_{1}$ and $L_{2}$ with the obvious identification of these lines with the two $\mathbb{P}^{1}$ 's. Series XIII no 1, corresponds to (a), Series XIII no 2, corresponds to (b) and Series XIII no 3 to (c).

Rohn also considers the situation where the ruled surface has a pair complex conjugated lines as double lines and produces a standard form and an example, namely model 4 of series XIII.

Rohn's normal form for other curves $E \subset \mathbb{P}^{1} \times \mathbb{P}^{1}$ of type $(2,2)$.

These normal forms are useful for $\$ 3.2 .2,3.2 .5,3.2 .6$ and 3.2.8. There are three cases:

(a) $E$ is irreducible and has a node,

(b) $E$ is irreducible and has a cusp and

(c) $E$ is reducible or is not reduced.

In the following we use the notation and the ideas of the proof of the theorem.

(a) The non singular locus of $E$ is, after a choice of a point $e_{0}$, the group $\mathbb{G}_{m}$. Let $\sigma$ denote the automorphism $x \mapsto-x$ and define $\tau_{a}(x)=a x$. The automorphisms of order 2 of $E$ are $\sigma \tau_{a}$ (any $a \in \mathbb{G}_{m}$ ) and $\tau_{-1}$. Dividing $E$ by the action of $\sigma \tau_{a}$ yields the quotient $\mathbb{P}^{1}$ and dividing by the action of $\tau_{-1}$ yields a rational curve with a double point. Thus the two projections $\mathrm{pr}_{i}: E \rightarrow \mathbb{P}^{1}$ correspond to order two elements $\sigma \tau_{a_{i}}$ for $i=1,2$ with $a_{1} \neq a_{2}$. The required automorphism $C$ of order two should satisfy $C \sigma \tau_{a_{1}}=\sigma \tau_{a_{2}} C$. There are two possibilities for $C$, namely $C=\sigma \tau_{c}$ with $c^{2}=a_{1} a_{2}$. Thus we find a symmetric embedding $E \subset \mathbb{P}^{1} \times \mathbb{P}^{1}$ for any algebraically closed field of characteristic $\neq 2$.

For $\mathbb{R}$ as base field, the situation is more complicated. Suppose that both lines, i.e., the two factors $\mathbb{P}^{1}$, and $E$ are defined over $\mathbb{R}$. We assume that the non singular locus $E^{*}$ has a real point $e_{0}$. There are two possibilities for $E^{*}(\mathbb{R})$, namely: (i) $\mathbb{G}_{m}(\mathbb{R})=\mathbb{R}^{*}$ and (ii) $\mathbb{R} / \mathbb{Z}$.

In case (i), one has to solve the equation $c^{2}=a_{1} a_{2}$ with $c \in \mathbb{R}^{*}$. If there is a solution, then one has a symmetric embedding $E \rightarrow \mathbb{P}^{1} \times \mathbb{P}^{1}$, defined over $\mathbb{R}$. In the opposite case, one makes an anti-symmetric embedding (by adding some minus signs). The two standard equations are

$$
a_{1} \lambda^{2} \mu^{2}+a_{2}\left(\lambda^{2} \pm \mu^{2}\right)+2 a_{3} \lambda \mu=0, \quad \text { with } \lambda=\frac{x_{1}}{x_{0}}, \mu=\frac{y_{1}}{y_{0}} .
$$

In case (ii), the automorphism of order two are the maps $f_{a}: x \mapsto-x+a($ any $a \in \mathbb{R} / \mathbb{Z})$ and $x \mapsto x+1 / 2$. The last automorphism is ruled out because it does not give a $\mathbb{P}^{1}$ as quotient. Now we have to solve $C f_{a_{1}}=f_{a_{2}} C$ for some order two automorphism $C$. The two solutions for $C$ are $f_{c}$ with $2 c=a_{1}+a_{2}$. There are two solutions for $c$ in $\mathbb{R} / \mathbb{Z}$ and therefore there is a symmetric embedding. The standard equation is

$$
a_{1} \lambda^{2} \mu^{2}+a_{2}\left(\lambda^{2}+\mu^{2}\right)+2 a_{3} \lambda \mu=0, \quad \text { with } \lambda=\frac{x_{1}}{x_{0}}, \mu=\frac{y_{1}}{y_{0}} .
$$

Finally, there is the possibility that the two lines form a conjugate pair over $\mathbb{R}$ (we do not work out the details here).

(b) The non singular locus $E^{*}$ of $E$ is isomorphic to the additive group $\mathbb{G}_{a}$. The automorphism of order two are $f_{a}: x \mapsto-x+a$ (any $a \in \mathbb{G}_{a}$ ). The equation $C f_{a_{1}}=f_{a_{2}} C$ (with $a_{1} \neq a_{2}$ ) has a unique solution $C=f_{c}$ with $2 c=a_{1}+a_{2}$. Thus there exists a symmetric embedding $E \subset \mathbb{P}^{1} \times \mathbb{P}^{1}$ and this embedding is unique. The above is valid for any field of characteristic $\neq 2$, because the group $\mathbb{G}_{a}$ has no forms. The standard equation is 


$$
\lambda^{2} \mu^{2}+(\lambda-\mu)^{2}-2 \lambda \mu(\lambda+\mu)=0 .
$$

(c) For a reducible or non reduced $E$, Rohn obtains the following standard equations

$$
(\lambda+\mu)^{2}+2 a \lambda \mu=0, \lambda^{2} \mu^{2} \pm(\lambda-\mu)^{2}=0,(\lambda-\mu)^{2}=0 .
$$

Acknowledgement The authors thank the referee for his very useful remarks.

Open Access This article is distributed under the terms of the Creative Commons Attribution Noncommercial License which permits any noncommercial use, distribution, and reproduction in any medium, provided the original author(s) and source are credited.

\section{References}

1. Barth, W., Peters, C., van der Ven, A.: Compact complex surfaces. Springer, Berlin (1984)

2. Bottema, O.: A classification of rational quartic ruled surfaces. Geometriae Dedicata 1(3), 349-355 (1973)

3. Cayley, A.: A third memoir on skew surfaces, otherwise scrolls. Philos. Trans. R. Soc. Lond. 159, 111126 (1869)

4. Chasles, M.: Sur les six droites qui peuvent être les directions de six forces en équilibre. Propriétés de l'hyperboloïde à une nappe et d'une certaine surface du quatrième ordre. Comptes Rendus Des Séances de l'Académie Des Sciences. Paris 52, 1094-1104 (1861)

5. Cremona, L.: Sulle Superficie Gobbe di Quarto Grado. Memorie dell' Accademia delle Science dell' Istituto di Bologna serie II, tomo VIII (1868), 235-250. Opere II, 420

6. Dolgachev, I.V.: Topics in Classical Algebraic Geometry, http://www.math.lsa.umich.edu/ idolga/ topics1.pdf

7. Edge, W.L.: The theory of ruled surfaces. Cambridge University Press, Cambridge (1931)

8. Hartshorne, R.: Algebraic geometry. Springer, Berlin (1977)

9. Meyer, W.: Fr. Flächen vierter und höherer Ordnung. Encyklopädie der Mathematischen Wissenschaften Mit Einschluss Ihrer Anwendungen IIIC 10b, 1744-1759 (1930)

10. Mohrmann, H.: Die Flächen vierter Ordnung mit gewundener Doppelkurve. Mathematische Annalen 89, 1-31 (1923)

11. Pascal, E.: Repertorium der Höheren Mathematik (Definitionen, Formeln, Theoreme, Literatur). II. Theil: die Geometrie. Teubner (1902)

12. Rohn, K.: Ueber die Flächen vierter Ordnung mit dreifachem Punkte. Mathematische Annalen 24(1), 55-151 (1884)

13. Rohn, K.: Die verschiedenen Arten der Regelflächen vierter Ordnung, (1886). Mathematische Abhandlungen aus dem Verlage Mathematischer Modelle von Martin Schilling. Halle a. S., 1904. Mathematische Annalen, vol. 28, No. 2, pp. 284-308 (1886)

14. Salmon, G.: A treatise on the analytic geometry of three dimensions. Hodges, Figgis, \& Co., Dublin (1882)

15. Schilling, M.: Catalog mathematischer Modelle für den höheren mathematischen Unterrich. Verlag von Martin Schilling, Leipzig (1911)

16. Segre, C.: Etude des différentes surfaces du 4 e ordre à conique double ou cuspidale (générale ou décomposée) considérées comme des projections de l'intersection de deux variétés quadratiques de l'espace à quatre dimensions. Mathematische Annalen 24(3), 313-444 (1884)

17. Sturm, R.: Die Gebilde ersten und zweiten Grades der Liniengeometrie in synthetischer Behandlung I. B.G. Teubner, Leipzig (1892)

18. Swinnerton-Dyer, H.P.F.: An enumeration of all varieties of degree 4. Am J Math 95(2), 403-418 (1973)

19. Urabe, T.: Dynkin graphs and combinations of singularities on quartic surfaces. Proc. Japan. Acad. Ser. A 61, 266-269 (1985)

20. Urabe, T.: Classification of non-normal quartic surfaces. Tokyo J Math 9(2), 265-295 (1986)

21. Urabe, T.: Elementary transformations of Dynkin graphs and singularities on quartic surfaces. Inventiones Mathematicae 87, 549-572 (1987)

22. Urabe, T.: Tie transformations of Dynkin graphs and singularities on quartic surfaces. Inventiones Mathematicae 100, 207-230 (1990)

23. Wong, B.C.: A study and classifications of ruled quartic surfaces by means of a point-to-line transformation. Univ. Calif. Publ. Math. 1(17), 371-387 (1923) 